\title{
EGFR signaling in colorectal cancer: a clinical perspective
}

This article was published in the following Dove Press journal:

Gastrointestinal Cancer:Targets and Therapy

19 January 2015

Number of times this article has been viewed

\section{Piercarlo Saletti ${ }^{1}$ \\ Francesca Molinari \\ Sara De Dosso' \\ Milo Frattini}

'Oncology Institute of Southern Switzerland, Bellinzona, ${ }^{2}$ Laboratory of Molecular Pathology, Institute of Pathology, Locarno, Switzerland
Correspondence: Milo Frattini Laboratory of Molecular Pathology, Institute of Pathology, 24 Via in Selva, Locarno 6600, Switzerland

$\mathrm{Tel}+41918160805$

Fax $+4|9| 8160719$

Email milo.frattini@ti.ch

\begin{abstract}
Colorectal cancer (CRC) remains a formidable health burden worldwide, with up to $50 \%$ of patients developing metastases during the course of their disease. This group of CRC patients, characterized by the worst prognosis, has been extensively investigated to improve their life expectancy. Main efforts, focused on the epidermal growth-factor receptor (EGFR), which plays a pivotal role in CRC pathogenesis, have led to the development and introduction in clinical practice of specific targeted therapies (ie, monoclonal antibodies). Subsequently, the scientific community has tried to identify molecular predictors of the efficacy of such therapies. However, it has become clear that EGFR alterations occurring in CRC are difficult to investigate, and therefore their predictive role is unclear. In contrast, the clinical role of two downstream members (KRAS and NRAS) has been clearly demonstrated. Currently, EGFRtargeted therapies can be administered only to patients with wild-type KRAS and NRAS genes. Our review addresses the medical management of metastatic CRC. Specifically, we describe in detail the molecular biology of metastatic CRC, focusing on the EGFR signaling pathway, and we discuss the role of current and emerging related biomarkers and therapies in this field. We also summarize the clinical evidence regarding anti-EGFR monoclonal antibodies and examine potential future perspectives.
\end{abstract}

Keywords: colorectal cancer, EGFR, gene mutations, cetuximab, panitumumab

\section{Introduction}

With 1.25 million patients diagnosed with the disease, colorectal cancer (CRC) remains a formidable health burden worldwide, accounting for more than 600,000 patient deaths every year. ${ }^{1,2}$ Approximately a quarter of patients present with synchronous metastases, and up to $50 \%$ of patients will develop metastases during the course of their disease. ${ }^{3}$ As a result of advances over the last 2 decades, overall survival (OS) may now be as long as approximately 30 months in patients with the poorest prognosis, characterized by metastatic disease (mCRC), with up to $70 \%$ of these patients receiving at least two treatment lines..$^{4-8}$ These achievements are attributed to the introduction of new chemotherapeutic agents, the incorporation of novel targeted therapies, and the expansion of indications for liver resection. In parallel, great effort is underway to shift from a "one size fits all" approach to more personalized medicine. Regarding this latter approach, a variety of prognostic (ie, information on the natural history of the patient's disease independent of treatments) and predictive (ie, information concerning the likelihood of response to a particular treatment) biomarkers are under evaluation.

Our review addresses the medical management of mCRC. Specifically, we describe the molecular biology of $\mathrm{mCRC}$, focusing on the epidermal growth factor receptor 
(EGFR) signaling pathway, and we discuss the role of current and emerging related biomarkers and therapies in this field. We also summarize the clinical evidence regarding antiEGFR monoclonal antibodies (mAbs) and examine potential future perspectives.

\section{Metastatic colorectal cancer: modern options in a continuum of care}

The initial consideration is whether the treatment goal is curative (immediately or leading to potentially resectable disease) or palliative, with the aims of prolonging survival and achieving symptom control and quality of life. ${ }^{3}$ By integrating systemic therapy and surgery in patients with limited liver metastases, 5-year OS ranges from $40 \%$ to $60 \% .^{9-11}$ The contribution of other locoregional treatments (radioembolization, chemoembolization, or stereotactic radiotherapy) in the treatment of liver metastases has not been fully elucidated, although upfront radiofrequency ablation with or without liver surgery followed by chemotherapy has been shown to improve progression-free survival (PFS) compared to chemotherapy alone for unresectable liver metastases. ${ }^{12}$ Therefore, these options may be considered in selected patients with chemorefractory liver disease. The management of mCRC involves various agents, given across a continuum of care. Interchangeable doublets (fluoropyrimidine and oxaliplatin [FOLFOX] or fluoropyrimidine and irinotecan [FOLFIRI], or their variations incorporating the oral fluoropyrimidine capecitabine) are recommended in rapidly progressing and/ or symptomatic disease, ${ }^{13,14}$ whereas the triplet FOLFIRINOX may be appropriate when maximizing tumor response may lead to secondary liver resection. ${ }^{15}$ To limit toxicities, a sequence of initial single-agent fluoropyrimidine followed by combination chemotherapy may be reasonable in low-burden, slowly progressing, and/or asymptomatic disease, particularly in elderly patients. ${ }^{16,17}$ The feasibility of chemotherapy-free intervals has been studied, and current data do not support the safety of a full chemotherapy holiday. In choosing oxaliplatin-based regimens, a stop-and-go strategy is feasible in responding patients, who continue on maintenance chemotherapy with or without a biological agent. ${ }^{18-20}$ In selected patients, observation may also be considered. ${ }^{21}$

The ability of targeted therapies to provide a survival benefit is now well established, although the improvement has been below expectations. In first-line treatment, the addition of the antiangiogenic bevacizumab to oxaliplatin- or irinotecan-based regimens improves PFS, and as observed with irinotecan only, OS compared with chemotherapy alone. ${ }^{2-24}$ Bevacizumab also improves OS in second-line therapy, and with a different chemotherapy backbone, beyond first progression. ${ }^{25,26}$ Moreover, the addition of bevacizumab to capecitabine significantly improves PFS, with a strong trend in OS and an acceptable toxicity profile among patients greater than 70 years of age. ${ }^{27}$ More recently, the recombinant fusion protein ziv-aflibercept (administered in second-line therapy, in combination with FOLFIRI) and the oral multikinase inhibitor regorafenib (in patients treated with all active drugs) have joined the treatment armamentarium, based on OS improvement. ${ }^{28,29}$ In this era of personalized medicine, the anti-EGFR mAbs cetuximab and panitumumab have significantly contributed to the development of more active therapeutic options.

\section{EGFR signaling and specific therapies}

The EGFR signaling pathway is believed to play a pivotal role in tumor growth and the progression of various cancers, mainly in solid tumors, including CRC. The EGFR gene, located on chromosome $7 \mathrm{p} 12-13$, encodes for a $170 \mathrm{kDa}$ transmembrane receptor comprising an extracellular ligandbinding domain and an intracellular tyrosine kinase (TK) domain. EGFR belongs to the ErbB family of receptor TKs (which includes ErbB1 [EGFR or HER1], ErbB2 [HER2], ErbB3 [HER3], and ErbB4 [HER4]). ${ }^{30}$ EGFR is activated by several ligands, including EGF, transforming growth factor- $\alpha$, amphiregulin (AREG), heparin-binding EGF, epiregulin (EREG), and betacellulin. Ligand binding induces receptor dimerization with another EGFR monomer (homodimerization) or with a monomer of another ErbB family member (heterodimerization). As a consequence, several tyrosine residues in the intracellular domain are phosphorylated, creating a series of high-affinity binding sites for various transducing molecules. The two main pathways activated by EGFR are the RAS-RAF-MAP kinase pathway and the PI3K-PTEN-Akt pathway. These pathways are involved in transmitting mitogenic signaling into the nucleus by regulating several transcription factors, which in turn control the expression of genes relevant for several cellular responses, such as cell proliferation, migration, differentiation, and apoptosis. ${ }^{31-35}$

EGFR is normally highly regulated by inhibitory mechanisms, including dephosphorylation by protein tyrosine phosphatases and de novo expression of EGFR inhibitors. ${ }^{36-41}$ Alterations of these regulation mechanisms (leading to an aberrantly high level of receptor activation and therefore to the constitutive activation of downstream signal-transduction pathways, causing the tumor growth to be strictly dependent 
on EGFR, a process also known as "tumor addiction") are tumorigenic, directly involve EGFR, and include hyperactivating mutations, protein overexpression, and gene amplification. Furthermore, the overexpression of receptor ligands and/or the loss of negative regulatory mechanisms are alternative and strong mechanisms of EGFR deregulation. ${ }^{34,42,43}$

In contrast to other cancer types, oncogenic mutations in the EGFR gene are rare in CRC. ${ }^{44-47}$ Rather, the principal mechanism of deregulation of EGFR in CRC is represented by protein overexpression (defined as $2+$ and/or 3+ staining or in $>50 \%$ of cells by immunohistochemical analyses) in $35 \%-50 \%$ of patients. ${ }^{42,48}$ Several studies have demonstrated a statistically significant association between EGFR overexpression and poor prognosis. ${ }^{49-53}$ However, other reports have failed to confirm this correlation, and therefore the prognostic role of EGFR deregulation remains elusive. ${ }^{48,54-56}$

\section{Targeting EGFR in CRC: anti-EGFR mAbs}

Given the important role of EGFR and its downstream pathways in tumorigenesis and disease progression, this receptor has become a relevant and promising target for anticancer therapies. In vitro and in vivo studies have demonstrated that blocking EGFR and downstream signaling may lead to carcinoma cell-growth inhibition, resulting in significant benefits for cancer patients. Several therapeutic approaches to targeting EGFR have been explored, most of which reached clinical testing. Two classes of EGFR antagonists have been developed, and are currently used in cancer treatment: mAbs, which prevent ligand binding to the receptors, and TK inhibitors, small molecules that compete for adenosine triphosphate binding to the TK domain of the receptor. Both approaches lead to the inhibition of EGFR autophosphorylation. ${ }^{57}$ Through the application of these agents to the treatment of tumors in which EGFR signaling plays a pivotal role, it has been demonstrated that TK-inhibitor (TKI) efficacy is restricted to cases carrying EGFR mutations (occurring in the TK domain encoded by exons 18-21, with the important exception of exon 20 mutations, which appear to be blocked only by irreversible TKIs), and therefore CRC patients in whom $E G F R$ mutations are very rarely detected cannot benefit from TKI administration. ${ }^{58}$

In contrast, mAbs have demonstrated promising results in the treatment of mCRC. Currently, two anti-EGFR mAbs have been approved by the US Food and Drug Administration and by the European Medicines Agency for the treatment of $\mathrm{mCRC}$, based on the improvement of PFS and OS when used as single agents or in combination with chemotherapy (detailed later). Cetuximab, a human-mouse chimeric $\operatorname{IgG}_{1}$
$\mathrm{mAb}$, was the first EGFR-targeted agent approved for the treatment of CRC. Panitumumab, a fully humanized $\operatorname{IgG}_{2}$ $\mathrm{mAb}$, was more recently approved in the US and Europe as third-line treatment of mCRC. ${ }^{58-60}$ Cetuximab and panitumumab display the same mechanism of action: they bind to the extracellular domain of EGFR, thus occluding the ligandbinding region and thereby blocking TK activation, inducing its internalization and degradation. ${ }^{58}$ Therefore, by inhibiting EGFR downstream pathways, they stimulate apoptosis. Additionally, anti-EGFR mAbs, particularly those of the $\mathrm{IgG}_{1}$ subclass, may recruit host immune mechanisms to attack the targeted cancer cell. These mechanisms include antibody-dependent cellular cytotoxicity, and to a lesser extent complement-mediated cytotoxicity. ${ }^{61,62}$ Irrespective of the anti-EGFR drug used, the clinical results of myriad studies have shown superimposable results for cetuximab and panitumumab.

The ability of cetuximab to block the EGFR pathway is supported by preclinical and clinical studies. At the preclinical level, it has been demonstrated that cetuximab alone primarily exhibits cytostatic activity, whereas its combination with other chemotherapeutic agents (such as platinum-derived compounds and irinotecan) potentiates the antitumoral activity of the individual therapies. ${ }^{63,64}$ One hypothesis for this synergy is that for the majority of cell lines, blocking EGFR signaling is insufficient for cytotoxicity, whereas EGFR inhibition may render the cells more vulnerable to chemotherapy. ${ }^{34}$

At the clinical level, cetuximab was the first mAb to demonstrate efficacy in CRC. Phase II trials demonstrated that patients with advanced CRC had a response rate (RR) of $11 \%$ when cetuximab was administered as single-agent therapy, and $23 \%$ when combined with irinotecan. Recently, panitumumab has been reported to produce similar results in a group of mCRC chemotherapy-refractory patients. ${ }^{6}$ EGFR mAbs have been evaluated as first-, second-, or third-line therapy, either as single agents or in combination with various chemotherapeutic molecules. Both antibodies have been shown to reduce the risk of tumor progression and to improve OS, PFS, and quality of life of patients with refractory CRC. ${ }^{59,65,66}$

Because only a subgroup of patients benefited from $\mathrm{mAb}$ administration, numerous retrospective and prospective studies conducted subsequently sought molecular predictors of anti-EGFR mAb efficacy. Currently, only RAS testing has been adopted in clinical practice, after extensive demonstration that the presence of $R A S$ mutations is significantly correlated with resistance to EGFR-targeted therapies. In the 
following sections, we summarize the predictive role played by the EGFR pathway in mCRC patients treated with cetuximab or panitumumab.

\section{Molecular mechanism of response and resistance to EGFR-targeted monoclonal antibodies \\ EGFR protein expression}

Regarding the predictive role of EGFR deregulation in mCRC patients treated with anti-EGFR mAbs, initially it was hypothesized that EGFR-targeted agents would be most effective in those tumors overexpressing the protein. However, it was promptly documented that the levels of EGFR protein expression detected by immunohistochemistry (IHC) were not correlated with clinical response..$^{35,65-67}$ In particular, in the study of Chung et al, ${ }^{67}$ four of $16(25 \%)$ patients with EGFR-negative tumors who received cetuximab-based therapy experienced a partial response. Subsequent retrospective analysis of multiple series and data from the PRIME trial confirmed these data, including in wild type (wt) KRAS tumors. ${ }^{68-70}$

The lack of association between EGFR protein expression by IHC and response to EGFR-targeted agents is likely due to many technical reasons. IHC is not a strictly quantitative method, and the choice of tissue fixative, the tumor tissuestorage time, the choice of primary antibody, and the lack of standardized evaluation criteria, together with the disparity between the form of the epitope of EGFR protein detected by IHC and that targeted by anti-EGFR mAbs, all represent potential pitfalls and have a substantial impact on the determination of EGFR immunoreactivity. ${ }^{71-73}$ Moreover, EGFR expression might differ between primary tumors and metastatic sites, and therefore the evaluation of EGFR expression in the primary tumor may be inappropriate for predicting the treatment response of metastases. Lastly, there is no correlation between EGFR protein expression and EGFR gene amplification. As a result of all of these considerations, cetuximab and panitumumab are now administered without the need for EGFR testing.

\section{EGFR gene amplification and copy number}

Recent studies on colon cancer have shown that a modest increase in EGFR gene copy number (three- to fivefold) is present in up to $50 \%$ of cases, and that this is caused mainly by polysomy rather than by gene amplification. However, it appears that increased EGFR gene copy number does not always translate into increased EGFR protein expression, ${ }^{74-76}$ in contrast to other ErbB family members. EGFR gene gain can be analyzed by fluorescent in situ hybridization (FISH), chromogenic ISH, or polymerase chain reaction-based methods, although the most frequently used method to assess EGFR gene status in CRC is FISH.

The predictive role of EGFR gene copy-number gain in $\mathrm{mCRC}$ patients treated with anti-EGFR mAbs has been demonstrated by different studies. Initial studies revealed that patients with tumors showing EGFR gene amplification or chromosome 7 polysomy responded to cetuximab therapy. ${ }^{77}$ Additional studies, also in large cohorts, confirmed these results, demonstrating that patients with fewer than three $E G F R$ gene copies per nucleus had a relatively low likelihood of response to EGFR-targeted $\mathrm{mAb}$ treatment. ${ }^{75,78-81}$ Conversely, only one study contradicted this evidence by showing a lack of statistical correlation between EGFR gene copy number and cetuximab treatment when the well-established FISH interpretation criteria used for non-small-cell lung cancer evaluation were applied. ${ }^{82}$ Thereafter, two studies (also in wt $K R A S$ patients) provided evidence anew regarding a statistically significant correlation between $E G F R$ gene copy number and RR, with a significant increase of the median time to progression in patients showing EGFR gain. ${ }^{83,84}$ However, all of the aforementioned studies applied different types of cutoffs to define $E G F R$ gene status. When comparative analyses performed on the same samples in different institutions showed a lack of reproducibility for $E G F R$ gene-status evaluation by FISH methodology, it became clear that EGFR gene status cannot be used in clinical settings to predict the efficacy of EGFR-targeted therapies either. ${ }^{85,86}$

In this context, it is important to highlight that the exact definition of the relationship between $E G F R$ gene status and the cetuximab/panitumumab response is complicated by the predictive role played by EGFR downstream effectors (discussed in "EGFR downstream effectors").

\section{EGFR ligands epiregulin and amphiregulin}

Independent groups recently reported in retrospective series of mCRC patients treated with cetuximab monotherapy or in combination with chemotherapy that increased expression of genes encoding two EGFR ligands - AREG and EREG strongly correlates with therapeutic benefit from cetuximab in wt $K R A S$ patients, both in terms of disease-control rate and PFS, whereas the impact on OS was not significant. ${ }^{87-91}$ However, similar to EGFR copy-number gain, due to the lack of standardization of the analytic method (real-time polymerase chain reaction), AREG and EREG expression levels are not routinely measured in clinical practice, and further evaluation of their role is required. 


\section{EGFR downstream effectors}

In addition to molecular alterations of the $E G F R$ gene and of its ligands, specific alterations of EGFR downstream effectors have been demonstrated to be linked with the response to anti-EGFR therapies.

EGFR downstream pathways alterations include oncogenic point mutations in the KRAS, NRAS, BRAF, and PIK3CA genes (reported in approximately $40 \%, 5 \%, 10 \%$, and $20 \%$ of CRC cases, respectively), and PTEN loss of function (due to mutations, allelic loss, or epigenetic alterations, cumulatively reported in approximately $20 \%-30 \%$ of cases), which occur independently of EGFR status (Figure 1). 5,75,77,79,82

\section{RAS}

The highly homologous family of $R A S$ oncogenes (KRAS, $H R A S$, and NRAS) encodes for guanosine diphosphate (GDP)/guanosine triphosphate (GTP)-binding proteins that act as self-inactivating intracellular signal transducers. RAS proteins normally cycle between active GTP-bound and inactive GDP-bound conformations. RAS mutations are one of the most common gene alterations in human cancer. ${ }^{92,93}$ Oncogenic RAS mutations result in RAS proteins that are permanently in the active GTP-bound form, thus leading to the constitutive activation of the MAPK pathway. Unlike wt RAS proteins that are inactivated after a short time, the mutated proteins are able to continuously activate signaling pathways

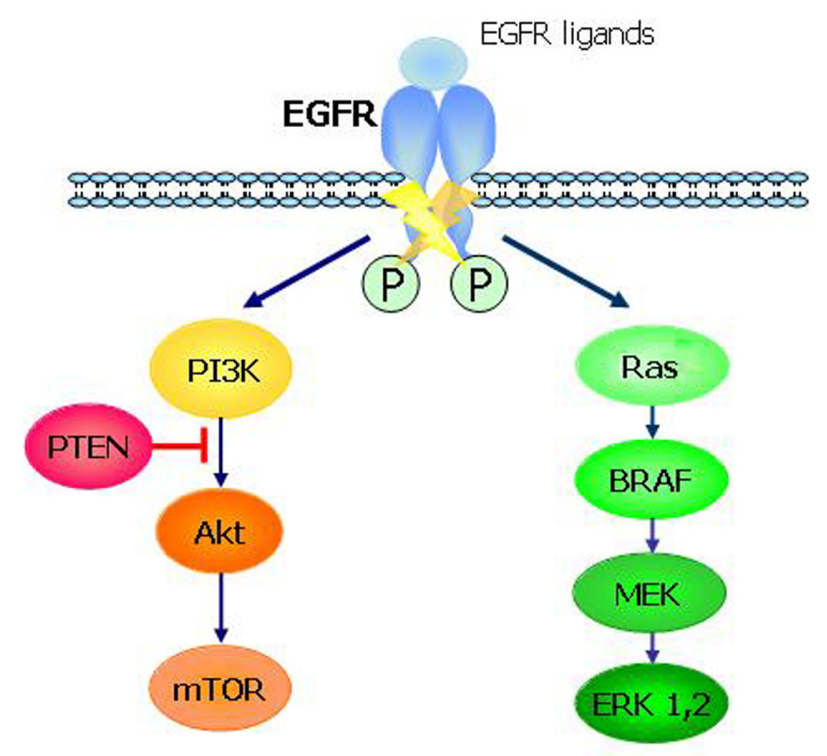

\section{Cell survival Apoptosis inhibition}

Cell proliferation

Figure I The EGFR pathway.

Abbreviation: EGFR, epidermal growth-factor receptor. in the absence of any upstream stimulation, including EGFR. More than $90 \%$ of KRAS mutations involve codons 12 and 13 (exon 2), with approximately $80 \%$ occurring in codon $12 .{ }^{94}$ Mutations in other exons ( 3 and 4 ) have also been reported; however, these comprise $<10 \%$ of mutations. ${ }^{93}$ A similar situation is observed for $N R A S$, although the most frequent change occurs at exon 3 (codon 61). Initially, KRAS mutations emerged as the main predictor of resistance to anti-EGFR mAbs. This fact has been consistently demonstrated in small single-arm data sets and also by retrospective analysis of large Phase III studies and in some prospective trials of patients receiving first or subsequent lines of treatment. In these studies, patients with mCRC harboring KRAS mutations did not show any benefit of treatment with cetuximab or panitumumab either alone or in combination with standard chemotherapy. ${ }^{35}$

Although all KRAS mutations display the same effect on KRAS protein activity, recent evidence appears to demonstrate that they may exert different effects on the efficacy of EGFR-targeted therapies. In fact, it has been proposed that patients with KRAS codon 13 mutations are not resistant to anti-EGFR mAbs, ${ }^{92}$ although this hypothesis was not confirmed by a pooled analysis of three randomized studies. ${ }^{95,96}$ As regards the predictive value of $K R A S$ exon 3 and 4 mutations, data obtained from retrospective studies ${ }^{92,97}$ demonstrated that these mutations share biological behavior with $K R A S$ codon 12 and 13 alterations, thus indicating that they may confer anti-EGFR $\mathrm{mAbs}$ resistance. These preliminary data have been confirmed by two large studies, which additionally demonstrated that NRAS mutations also display the same negative effect on the efficacy of EGFR-targeted therapies. ${ }^{95,96}$ Presently, therefore, it is mandatory to evaluate the molecular status of the KRAS and NRAS genes before the administration of EGFR-targeted therapies.

\section{BRAF}

The BRAF gene, located on chromosome 7, encodes for an RAS effector belonging to the RAF family of Ser-Thr kinase proteins. The $B R A F$ gene product is recruited to the plasma membrane upon binding to GTP-bound RAS, and represents a key point in the signal transduction through the MAPK pathway. BRAF is the only RAF protein found to be frequently mutated in cancer. ${ }^{98}$ In CRC, $B R A F$ mutations, occurring in approximately $10 \%$ of cases, are all represented by V600E amino acid substitution, and have been detected only in wt KRAS cases. ${ }^{99}$ These mutual exclusions have led to the assumption that $B R A F$ and $K R A S$ alterations might have the same functional effect in 
colorectal carcinogenesis, although mutated BRAF protein has a 50-fold-lower transforming activity than mutated RAS proteins. ${ }^{100}$ Therefore, the predictive role of $B R A F$ mutations has been evaluated by several retrospective studies, most of which showed a significant association between the presence of $B R A F$ mutations and resistance to anti-EGFR mAbs in mCRC patients refractory to standard chemotherapy. ${ }^{92,101,102}$

However, the results from patients treated with anti-EGFR mAbs in first-line therapy have shown that $B R A F$-mutant patients experienced some benefit from the addition of cetuximab. ${ }^{103}$ Considering that $B R A F$ mutations are an established prognostic factor linked with adverse outcome, it is difficult to clarify whether BRAF also has a predictive value, as BRAF prognostic value may override its predictive role. ${ }^{5,103}$ Therefore, the role of the $B R A F$ mutation as a negative predictor of cetuximab efficacy has yet to be clarified.

\section{PIK3CA}

$\mathrm{PI} 3 \mathrm{Ks}$ are heterodimeric lipid/protein kinases that differ in structure, substrate specificity, tissue distribution, function, and mechanisms of activation and regulation. ${ }^{104}$ Activation of PI3Ks stimulates various downstream pathways involved in the regulation of several cellular functions, including cellular growth, transformation, adhesion, apoptosis, survival, and motility. ${ }^{104}$ PI3Ks are antagonized by the phosphatase PTEN, which catalyzes the opposite reaction. Constitutive activation and overexpression of PI3Ks (and inactivation of PTEN) results in enhanced PI3K signaling, leading to oncogenic cellular transformation and cancer. Only PI3K proteins that contain the catalytic subunit $\mathrm{p} 110 \alpha$ and its associated regulatory subunit p85 (belonging to the class IA) are involved in tumorigenesis, as only the $P I K 3 C A$ gene, encoding for the $\mathrm{p} 110 \alpha$ subunit, has been found to be mutated in several tumors. ${ }^{104}$

The predictive effect of PIK3CA mutations in mCRC patients treated with EGFR-targeted therapies has been assessed by few studies. By analyzing CRC cellular models, it has been demonstrated that cell lines harboring PIK3CA mutations (as well as PTEN-null phenotype) are significantly more resistant to cetuximab compared with $P I K 3 C A / \mathrm{PTEN}$ normal cell lines. ${ }^{105}$ These results were confirmed at a clinical level by large retrospective studies; however, to date it appears that only PIK3CA mutations occurring at exon 20 play a negative predictive role for EGFR-targeted therapies. ${ }^{92,106-108}$ Overall, these data appear to indicate that specific PIK3CA mutations on exon 20 may be associated with resistance to
EGFR-targeted therapies. However, as $P I K 3 C A$ mutations may coexist with $K R A S$ and $B R A F$ mutations, it is more challenging to ascertain their clinical significance, and therefore larger studies confirming this hypothesis are required.

\section{PTEN}

$P T E N$ is a tumor-suppressor gene that encodes for a 403-amino acid protein counteracting PI3K activity. ${ }^{109}$ In CRC, PTEN is altered through mixed genetic/epigenetic mechanisms (intragenic mutation/epigenetic or 10q23 loss of heterozygosity/epigenetic), which lead to the biallelic inactivation of the protein in $20 \%-30 \%$ of cases. In addition to PTEN loss of heterozygosity and mutations, PTEN promoter hypermethylation is a frequent event in sporadic microsatellite unstable CRC, and may constitute an important epigenetic mechanism of PTEN inactivation in this setting. Therefore, because all of these alterations lead to decreased protein expression, the preferred method to evaluate PTEN inactivation is represented by IHC, which is however associated with reproducibility concerns. ${ }^{35}$

In fact, the preliminary studies showing PTEN loss of expression as a novel mechanism of resistance to EGFR-targeted therapies have not been confirmed, and consequently PTEN evaluation warrants more extensive investigation in large studies..$^{70,79,83,107,110-112}$

\section{Anti-EGFR mAbs: clinical perspective}

The choice of first-line treatment for patients with $\mathrm{mCRC}$ is based on tumor- and patient-related factors and molecular information to determine the individual treatment aim and intensity. Recent advances (ie, extended $R A S$ testing) enable tailored patient assignment to the most beneficial treatment approach.

\section{Unresectable metastatic disease Upfront therapy}

Several clinical trials have shown the efficacy of anti-EGFR $\mathrm{mAbs}$ in combination with chemotherapy in treating $\mathrm{mCRC}$, irrespective of the age of patients included (Table 1).

\section{Cetuximab}

In the CRYSTAL trial, 1,198 untreated patients with mCRC were randomized to FOLFIRI alone or in combination with cetuximab. ${ }^{5,113}$ In the intent-to-treat (ITT) population, PFS (the primary end point) significantly improved in the cetuximab arm (8.9 versus 8.0 months, hazard ratio [HR 0.85]; $P=0.048$ ), but not OS. However, for patients who exhibited 
Table I Anti-EGFR mAbs as first-line therapy

\begin{tabular}{|c|c|c|c|c|c|c|c|c|c|}
\hline Study & $\begin{array}{l}\text { Treatment } \\
\text { arms }\end{array}$ & $\begin{array}{l}\text { Primary } \\
\text { end point }\end{array}$ & Population & $\begin{array}{l}\text { Patients, } \\
\text { n }\end{array}$ & $\begin{array}{l}\text { Median PFS, } \\
\text { months }\end{array}$ & $\begin{array}{l}\text { HR } \\
P \text {-value }\end{array}$ & $\begin{array}{l}\text { Median OS, } \\
\text { months }\end{array}$ & $\begin{array}{l}\text { HR } \\
P \text {-value }\end{array}$ & $\begin{array}{l}\text { RR, \% } \\
\text { P-value }\end{array}$ \\
\hline \multicolumn{10}{|l|}{ Cetuximab } \\
\hline Van Cutsem & FOLFIRI + C & PFS & ITT & 1,198 & 8.9 & 0.85 & 19.9 & 0.93 & 46.9 \\
\hline \multirow[t]{8}{*}{ et $\mathrm{al}^{113}$} & FOLFIRI & & & & 8.0 & $P=0.048$ & 18.6 & $P=0.31$ & 38.7 \\
\hline & & & & & & & & & $P=0.0038$ \\
\hline & & & KRAS wt & 666 & 9.9 & 0.696 & 23.5 & 0.796 & 57.3 \\
\hline & & & & & 8.4 & $P=0.0012$ & 20.0 & $P=0.0093$ & 39.7 \\
\hline & & & & & & & & & $P<0.001$ \\
\hline & & & KRAS mut & 397 & 7.4 & 1.171 & 16.2 & 1.035 & 31.3 \\
\hline & & & & & 7.7 & $P=0.26$ & 16.7 & $P=0.75$ & 36.1 \\
\hline & & & & & & & & & $P=0.35$ \\
\hline Maughan & FOLFOX/XELOX + C & OS & KRAS wt & 729 & 8.6 & 0.96 & 17.0 & 1.04 & 64 \\
\hline \multirow[t]{4}{*}{ et $\mathrm{al}^{70}$} & FOLFOX/XELOX & & & & 8.6 & $P=0.60$ & 17.9 & $P=0.68$ & 57.0 \\
\hline & & & & & & & & & $P=0.049$ \\
\hline & & & KRAS mut & 565 & & & 13.6 & 0.98 & \\
\hline & & & & & & & 14.8 & $P=0.80$ & \\
\hline \multirow[t]{13}{*}{ Tveit et al ${ }^{116}$} & $\mathrm{FLOX}+\mathrm{C}$ & PFS & ITT & 194 & 8.3 & 0.89 & 19.7 & 1.06 & 49.0 \\
\hline & Intermittent FLOX $+\mathrm{C}$ & & & 187 & 7.3 & $P=0.31$ & 20.3 & $P=0.67$ & $P=0.15$ \\
\hline & FLOX & & & 185 & 7.9 & NR & 20.4 & 1.03 & 47.0 \\
\hline & & & & & & & & $P=0.79$ & 41.0 \\
\hline & & & KRAS wt & 97 & 7.9 & 1.07 & 20.1 & 1.14 & 46.0 \\
\hline & & & & 109 & 7.5 & $P=0.66$ & 21.4 & $P=0.48$ & $P=0.89$ \\
\hline & & & & 97 & 8.7 & NR & 22.0 & 1.08 & 51.0 \\
\hline & & & & & & & & $P=0.66$ & $P=0.89$ \\
\hline & & & & & & & & & 47.0 \\
\hline & & & KRAS mut & 72 & 9.2 & 0.71 & 21.1 & 1.03 & 49.0 \\
\hline & & & & 65 & 7.2 & $P=0.07$ & 20.5 & $P=0.89$ & $P=0.31$ \\
\hline & & & & & 7.8 & NR & 20.4 & 1.04 & 42.0 \\
\hline & & & & & & & & $P=0.84$ & 40.0 \\
\hline Bokemeyer & FOLFOX $+\mathrm{C}$ & $\mathrm{RR}$ & ITT & 337 & 7.2 & 0.931 & 18.3 & 1.015 & 46.0 \\
\hline \multirow[t]{8}{*}{ et $\mathrm{al}^{114}$} & FOLFOX & & & & 7.2 & $P=0.62$ & 18.0 & $P=0.91$ & 36.0 \\
\hline & & & & & & & & & $P=0.064$ \\
\hline & & & KRAS wt & 179 & 8.3 & 0.567 & 22.8 & 0.855 & 57.0 \\
\hline & & & & & 7.2 & $P=0.0064$ & 18.5 & $P=0.39$ & 34.0 \\
\hline & & & & & & & & & $P=0.0027$ \\
\hline & & & KRAS mut & 136 & 5.5 & 1.72 & 13.4 & 1.290 & 34.0 \\
\hline & & & & & 8.6 & $P=0.0153$ & 17.5 & $P=0.20$ & 53.0 \\
\hline & & & & & & & & & $P=0.029$ \\
\hline \multicolumn{10}{|c|}{ Panitumumab } \\
\hline Douillard & FOLFOX $+\mathrm{P}$ & PFS & KRAS wt & 656 & 9.6 & 0.80 & 23.9 & 0.83 & 55.0 \\
\hline \multirow[t]{4}{*}{ et $a l^{6}$} & FOLFOX & & & & 8.0 & $P=0.02$ & 19.7 & $P=0.072$ & 48.0 \\
\hline & & & & & & & & & $P=0.068$ \\
\hline & & & KRAS mut & 440 & 7.3 & 1.29 & 15.5 & 1.24 & 40.0 \\
\hline & & & & & 8.8 & $P=0.02$ & 19.3 & $P=0.068$ & 40.0 \\
\hline Douillard & FOLFOX $+\mathrm{P}$ & PFS & RAS wt & 512 & 10.1 & 0.72 & 25.8 & 0.77 & NR \\
\hline \multirow[t]{3}{*}{ et $\mathrm{al}^{95}$} & FOLFOX & & & & 7.9 & $P=0.004$ & 20.2 & $P=0.009$ & \\
\hline & & & RAS mut & 548 & 7.3 & 1.31 & 15.5 & 1.21 & NR \\
\hline & & & & & 8.7 & $P=0.008$ & 18.7 & $P=0.04$ & \\
\hline
\end{tabular}

Abbreviations: C, cetuximab; HR, hazard ratio; EGFR, epidermal growth-factor receptor; FLOX, fluoropyrimidine + folinic acid + oxaliplatin; FOLFIRI, fluoropyrimidine + irinotecan; FOLFOX, fluoropyrimidine + oxaliplatin; ITT, intent to treat; mAbs, monoclonal antibodies; mut, mutant; NR, not reported; OS, overall survival; P, panitumumab; PFS, progression-free survival; RR, response rate; wt, wild type; XELOX, capecitabine + oxaliplatin.

wt $K R A S$, a greater and statistically significant benefit was observed for both PFS (9.9 versus 8.7 months, HR 0.68) and OS (23.5 versus 20.0 months, HR 0.796; $P=0.0093$ ). Cetuximab has also been evaluated in combination with FOLFOX in the randomized Phase II OPUS trial. ${ }^{114,115}$
In patients with wt $K R A S$, the combination significantly improved PFS ( 8.3 versus 7.2 months, HR $0.567 ; P=0.0064$ ), without benefit to OS (22.8 versus 18.5 months, HR 0.855 ; $P=0.39$ ). If cetuximab is combined with an oxaliplatinbased chemotherapy backbone, infusional 5-fluorouracil 
(5-FU) is preferable to an oral (XELOX) or bolus (FLOX) fluoropyrimidine-containing regimen. In fact, in the COIN trial, ${ }^{69}$ although the RR was superior in the experimental arm (64\% versus 57\%, $P=0.049)$, neither OS (primary end point; 17.0 versus 17.9 months, HR $1.04 ; P=0.67$ ) nor PFS (8.6 months in both arms, HR 0.96; $P=0.60)$ improved by adding cetuximab to oxaliplatin-based chemotherapy. Similarly, in the NORDIC VII trial, ${ }^{116}$ patients with wt $K R A S$ derived no benefit from cetuximab plus FLOX in terms of PFS (primary end point; 8.7 versus 7.9 months, HR 1.07; $P=0.66$ ) or OS (22.0 versus 20.1 months, HR $1.14 ; P=0.48$ ). In comparison with the OPUS trial, in which infusional 5-FU was administered in combination with oxaliplatin, the different fluoropyrimidine (capecitabine or bolus 5-FU) schedules used in these two trials in combination with oxaliplatin might explain the negative outcomes.

\section{Panitumumab}

In first-line treatment, panitumumab has been evaluated in a randomized trial in combination with FOLFOX. Retrospective analyses of the PRIME study clearly demonstrated the negative predictive value of KRAS mutation in exons 3 and 4 and NRAS mutations in exons 2, 3, and 4 in patients treated with panitumumab and FOLFOX. ${ }^{95}$ In patients with any $R A S$ mutation, the addition of panitumumab to FOLFOX had a detrimental effect on PFS (7.3 versus 8.7 months, HR $1.31 ; P=0.008)$ and OS (15.5 versus 18.7 , HR $1.21 ; P=0.040)$. In contrast, in 512 patients with tumors characterized by all wt $R A S$ genes, both PFS (primary end point; 10.1 versus 7.9 months, HR $0.72 ; P=0.004$ ) and OS (26.0 versus 20.2 months, HR $0.78 ; P=0.04$ ) were significantly in favor of the combination.

\section{Head to head with bevacizumab}

In the randomized Phase III AIO KRK-0306 (FIRE-3) study, ${ }^{117}$ FOLFIRI was evaluated either with cetuximab or bevacizumab in $592 \mathrm{wt} K R A S$ patients. In the ITT population, no difference in RR (primary end point; $62 \%$ versus $58 \%$, odds ratio $1.249 ; P=0.183$ ) was observed between the study arms. In contrast, a significant advantage in favor of the cetuximabcontaining arm was reported (72\% versus $63 \%, P=0.017)$. While PFS were superimposable (10 versus 10.3 months, HR $1.06 ; P=0.547)$, OS was significantly better in the cetuximab arm (28.7 versus 25 months, HR 0.77; $P=0.017$ ). Recent analyses demonstrated a more pronounced OS benefit in wt $R A S$ patients (33.1 versus 25.6 months, HR $0.70 ; P=0.011$ ) favoring the cetuximab arm. ${ }^{118}$ In this trial, the authors retrospectively evaluated the outcome of second-line therapies. The study recommended FOLFOX plus bevacizumab or irinotecan plus cetuximab according to the randomization arm, but clinicians could choose any second-line regimen. First-line PFS according to second-line antibody use was 9.2 months for anti-vascular endothelial growth factor (VEGF), 9.7 months for anti-EGFR mAbs, and 11.3 months for no mAbs, respectively $(P=0.001)$. Correspondingly, OS was 25.2 months for anti-VEGF, 23.7 months for anti-EGFR, and 30.8 months for no mAbs $(P=0.02)$. OS according to oxaliplatin use was 27.1 months for oxaliplatin versus 29.1 months for no oxaliplatin $(P=0.10)$. In the recently published randomized Phase II PEAK study, ${ }^{119}$ FOLFOX was evaluated either in combination with panitumumab or bevacizumab in 285 previously untreated wt $K R A S$ patients. In the ITT group, PFS (primary end point; 10.9 versus 10.1 months, HR $0.87 ; P=0.353$ ) was similar between the study arms, whereas OS was superior in the panitumumab arm (34.2 versus 24.3 months, HR $0.62 ; P=0.009)$. In the subgroup with all wt $R A S$ genes, the panitumumab arm was superior in terms of PFS (13.0 versus 9.5 months, HR $0.65 ; P=0.029)$ and OS (41.3 versus 28.9 months, HR $0.63 ; P=0.058$ ). Therefore, the similar results in both the FIRE-3 and PEAK trials suggest the beneficial impact of anti-EGFR mAbs plus chemotherapy in patients with all-wt $R A S$ genes.

However, conflicting results arose from the large CALGB/ SWOG 80405 trial. ${ }^{120}$ Previously untreated patients with wt $K R A S$ mCRC were randomized to receive either bevacizumab or cetuximab in combination with chemotherapy (FOLFOX or FOLFIRI, by investigator choice). Surprisingly, no differences in either OS (primary end point; 29 versus 29.9 months, HR $0.92 ; P=0.34$ ) or PFS (10.8 versus 10.4 months, HR $1.04 ; P=0.55)$ were observed between the treatment arms. Nonetheless, it is expected that expanded $R A S$ testing may identify subsets of patients who derive benefit from specific regimens. In anticipation of this possibility, the current evidence enhances the positioning of anti-EGFR mAbs in the first-line treatment of $\mathrm{mCRC}$.

\section{Secondary resectability}

In some patients, the achievement of a disease-free status, after downsizing by induction systemic therapy enabling secondary surgery, is the only means of conferring the potential of long-term survival or even cure. For this purpose, the most active induction chemotherapy should be used up-front, considering that early tumor shrinkage is associated with better outcome. ${ }^{121,122}$ A chemotherapy doublet with antiEGFR mAbs is an attractive option, as it can lead to higher $\mathrm{RR}$ and resectability rates in patients with wt $K R A S$ and initially unresectable liver-limited metastases compared to chemotherapy alone. In the updated analysis of the CRYSTAL 
trial, overall RR $(57.3 \%$ versus $39.7 \%, P<0.001)$, the rates of surgery for metastasis $(7.9 \%$ versus $4.6 \%, P=0.0633)$ and $\mathrm{R} 0$ resection $(5.1 \%$ versus $2.0 \%, P=0.0265)$ were significantly higher in the cetuximab arm. ${ }^{5}$ In the CELIM trial, the patients were randomized to receive cetuximab either with FOLFOX6 or FOLFIRI. ${ }^{123}$ In patients with wt KRAS tumors, an RR of $70 \%$ was reported along with a $34 \%$ R0 resection rate of liver metastases. Similar trends have been reported in the randomized Phase II OPUS trial, with an observed higher RR ( $57 \%$ versus $34 \%, P=0.0027$ ) in favor of cetuximab plus FOLFOX. ${ }^{115}$ In a recently published trial, cetuximab plus chemotherapy was compared with chemotherapy alone (FOLFOX6 or FOLFIRI) in patients with wt $K R A S$ unresectable liver-limited metastases. ${ }^{124}$ The combination arm demonstrated significantly improved conversion to resection (primary end point; $28.6 \%$ versus $13.2 \%, P=0.027$ ), R0 resection $(25.7 \%$ versus $7.4 \%, P=0.004)$, and RR $(57.1 \%$ versus $29.4 \%, P=0.001$ ), respectively. Lastly, cetuximab was also evaluated in combination with the triplet FOLFOXIRI in a Phase II trial of patients with wt KRAS mCRC who were younger than 70 years and with $0-1$ performance status. ${ }^{125}$ The overall RR was 70\%, and secondary R0 resections were performed in $37 \%$ of patients. In the final report of the PRIME trial, overall RR favored panitumumab plus FOLFOX4 (57\% versus $48 \%, P=0.02$ ) compared to chemotherapy alone in patients with the wt KRAS gene. ${ }^{126}$ Panitumumab was also evaluated in combination with FOLFOXIRI in patients with quadruple wt (KRAS, NRAS, HRAS, BRAF)-status mCRC. ${ }^{127}$ The objective RR was $89 \%$, conversion surgery was possible in $43 \%$, and $\mathrm{R} 0$ resection occurred in $35 \%$ of cases.

\section{Immediately resectable metastatic disease}

In patients with resectable disease, perioperative chemotherapy is an acceptable option considering the improvement in PFS at 3 years compared to surgery alone (35.4\% versus $28.1 \%$, HR $0.79 ; P=0.058) .{ }^{128}$ The New EPOC study evaluated the benefit of cetuximab in addition to FOLFOX in patients with wt KRAS operable liver metastases. ${ }^{129}$ PFS, the primary end point, was significantly shorter in the combination arm compared to chemotherapy alone (14.1 versus 20.5 months, HR 1.48; $P=0.030$ ). In view of this unexpected result, the addition of cetuximab to chemotherapy cannot be recommended in immediately resectable liver metastases.

\section{Anti-EGFR mAbs beyond progression}

Unlike the benefit of using bevacizumab beyond disease progression, ${ }^{130}$ data regarding anti-EGFR mAbs in this setting are relatively limited. The rate of poststudy use of
anti-EGFR mAb therapy varies from $6 \%$ to $12 \% .{ }^{113,116,126,131}$ In the FIRE-3 trial, $48.2 \%$ of patients in the cetuximab arm received second-line therapy including bevacizumab, whereas $14.4 \%$ continued on cetuximab. ${ }^{117}$ The post hoc exploratory analysis of the EPIC study suggested a potential clinical benefit of using cetuximab beyond disease progression. ${ }^{132}$ In fact, patients in the cetuximab-plus-irinotecan arm who went on to receive cetuximab in the poststudy-therapy phase had a median survival of 16.2 months, whereas those who did not receive any poststudy therapy and those who received poststudy therapy without cetuximab had median survival of 6.31 months and 13.0 months, respectively. With respect to the selection bias inherent to this unplanned analysis, the results should be regarded as hypothesis-generating. This issue is currently being explored in the ongoing CAPRI trial, ${ }^{132}$ in which wt $K R A S$ patients refractory to cetuximab plus FOLFIRI are randomized to receive FOLFOX alone or in combination with cetuximab.

\section{Second- and third-line therapies}

The EPIC trial was designed to assess whether the addition of cetuximab to irinotecan as second-line therapy would prolong OS in patients who failed upfront oxaliplatin and 5-FU. ${ }^{133}$ No improvement in OS was observed; however, patients in the combination arm had significantly longer PFS (4.0 versus 2.6 months, HR 0.692; $P<0.0001$ ) and higher RR (16.4\% versus $4.2 \%, P<0.0001)$. In a randomized Phase III trial, panitumumab was evaluated in second-line therapy in combination with FOLFIRI in 1,186 patients, and $K R A S$ status was available in $91 \%$ of them. ${ }^{131}$ In the wt KRAS subgroup, although PFS was significantly prolonged in the combination arm compared to FOLFIRI (3.9 versus 5.9 months, HR $0.73 ; P=0.004$ ), no significant difference in OS was observed (14.5 versus 12.5 months, HR $0.85 ; P=0.12$ ). Three randomized trials were conducted to evaluate the efficacy of anti-EGFR mAbs in third-line treatment or beyond. In the pivotal BOND trial, ${ }^{66} 329$ patients who progressed to an irinotecan-based regimen were randomized to receive either cetuximab and irinotecan or cetuximab alone. The RR was significantly higher in the combination arm $(22.9 \%$ versus $10.8 \%, P=0.007)$. The time to progression was also significantly prolonged in favor of the combination (4.1 versus 1.5 months, HR 0.54 ; $P<0.001$ ), whereas no difference in OS was observed. The NCIC CO.17 trial compared cetuximab to best supportive care (BSC) in 572 patients who had failed or had contraindications to all active chemotherapeutic agents. ${ }^{134}$ The OS improved in the cetuximab arm (6.1 versus 4.6 months, HR $0.77 ; P=0.005)$, and the grade of cutaneous rash 
strongly correlated with OS (no rash, 2.6 months; grade 1 rash, 4.8 months; grade 2 rash; 8.4 months; $P<0.001$ ). Lastly, KRAS status was retrospectively evaluated in $69 \%$ of patients and in wt KRAS patients; PFS, OS, and RR significantly improved with cetuximab over BSC. In the NCIC CO.17 study, panitumumab compared to BSC was evaluated in 463 chemorefractory patients with a preplanned analysis of $K R A S$ status. ${ }^{135}$ The PFS (primary end point; 8.0 versus 7.3 weeks, HR $0.54 ; P<0.0001$ ) was significantly prolonged in the panitumumab arm, whereas no difference in OS was reported, most likely due to the crossover after progression in the BSC group. In patients with wt KRAS status, PFS significantly increased (12.3 versus 7.3 weeks, HR 0.45), although again no differences in OS were detected in either of the KRAS subgroups. The PICCOLO trial was originally designed to evaluate panitumumab plus irinotecan compared to irinotecan alone in molecularly unselected patients refractory to a fluoropyrimidine with or without oxaliplatin. ${ }^{102}$ The study was amended to a prospectively stratified design, restricting panitumumab randomization to wt $K R A S$ patients. The OS primary end point was unmet (10.4 versus 10.9 months, HR 1.01; $P=0.91)$; however, longer PFS (HR $0.78, P=0.015)$ and RR (34\% versus $12 \%$, $P<0.0001)$ were noted in the combination arm. In the recently published ASPECCT trial, wt KRAS chemorefractory patients were randomized to receive panitumumab or cetuximab. ${ }^{136}$ The primary end point was OS, assessed for noninferiority. Panitumumab was noninferior to cetuximab $(P=0.0007)$, and both agents provided similar OS (10.4 versus 10.0 months, HR 0.97).

\section{Is the rechallenge of anti-EGFR mAbs effective?}

The strategy of rechallenge with anti-EGFR mAbs might be attractive, because $K R A S$ status remains largely unaltered during tumor progression. ${ }^{137,138}$ Preliminary evidence for this strategy was shown in wt $K R A S$ patients who had previously benefited from these drugs. In a Phase II study, ${ }^{139}$ 39 patients were retreated with cetuximab-based therapy after a new line of chemotherapy. The overall RR was $53.8 \%$ (including two complete responses), and PFS was 6.6 months. In another trial of patients treated with panitumumab after cetuximab-based therapy, an RR of $54.5 \%$ with additional stable disease in $18.2 \%$ was reported. ${ }^{140}$ Therefore, it appears plausible that some wt $R A S$ patients who have benefited initially from anti-EGFR based regimens might benefit from rechallenge, although more data are warranted. Conversely, there is no evidence to support switching to either cetuximab or panitumumab after failure of the other drug. ${ }^{140}$

\section{Anti-EGFR concurrent with anti-VEGF mAbs}

A close relationship of the VEGF and EGFR signaling cascades has been demonstrated in preclinical studies, and thus the combination of both anti-VEGF and anti-EGFR mAbs was evaluated as a biologically plausible and attractive strategy. ${ }^{141-144}$ In the randomized Phase II BOND-2 study, the benefit of bevacizumab plus cetuximab plus irinotecan or bevacizumab plus cetuximab alone was explored in irinotecan-refractory patients. ${ }^{145}$ The time to progression (7.3 versus 4.9 months), RR (37\% versus 20\%), and OS (14.5 versus 11.4 months) favored the triplet including both biologicals. Based on these promising data, 755 previously untreated patients were enrolled in the CAIRO2 trial to explore the efficacy of cetuximab added to bevacizumab, capecitabine and oxaliplatin. ${ }^{16}$ Unfortunately, the addition of cetuximab led to significantly shorter PFS (primary end point; 9.4 versus 10.7 months, HR $1.22 ; P=0.01$ ), whereas OS and RR were not significantly different. In the subgroup analysis according to KRAS status, the addition of cetuximab in the mutant subgroup led to worse PFS. The randomized Phase IIIB PACCE trial evaluated the efficacy of bevacizumab and chemotherapy, either oxaliplatin- or irinotecan-based, with or without panitumumab in previously untreated patients. ${ }^{146}$ In the final analysis, PFS decreased by 1.4 months in the panitumumab arm compared to the control arm (10.0 versus 11.4 months, HR 1.27). Subgroup analysis by KRAS status also demonstrated worse outcomes in both the wt and mutant subgroups in the panitumumab group. Based on these two large trials, the combination of anti-EGFR and anti-VEGF $\mathrm{mAbs}$ is not recommended.

\section{Maintenance}

After induction chemotherapy, maintenance is often offered to improve the duration of disease control, according to the OPTIMOX $2^{19}$ and $\mathrm{COIN}^{147}$ results. Final CAIRO3 results established the survival benefit of maintenance with bevacizumab plus capecitabine after first-line induction treatment. ${ }^{20}$ The potential role of anti-EGFR mAbs in maintenance was evaluated in the NORDIC-VII study, in which patients were randomly assigned to receive FLOX, cetuximab plus FLOX, or cetuximab plus intermittent FLOX. ${ }^{116}$ The OS (20.4 versus 19.7 versus 20.3 months, respectively) was almost identical in all three groups, suggesting that maintenance therapy with cetuximab might be feasible. 
The randomized Phase II COIN-B trial was designed as an exploratory, hypothesis-generating study to complement COIN, and patients were assigned to intermittent FOLFOX plus intermittent cetuximab versus continuous cetuximab. Both failure-free survival at 10 months (primary end point; $52 \%$ versus $50 \%$ ) and median failure-free survival (14.3 versus 12.2 months) favored planned maintenance with continuous cetuximab. ${ }^{148}$

\section{Future directions}

Extensive preclinical work on the potential mechanisms of resistance to EGFR inhibitors has guided the development of more efficient anti-EGFR mAbs, targeting simultaneously different receptors and other members of the EGFR/HER family, and combination strategies with agents targeting other receptors/proteins and downstream effectors.

\section{More efficient anti-EGFR mAbs}

The first approach to overcome resistance to anti-EGFR drugs is the development of mAbs with more efficient binding ability. Thus far, the most promising agent is Sym004, a new compound that combines two mAbs, which can bind simultaneously to two nonoverlapping epitopes on domain III of the extracellular domain of EGFR, inducing highly efficient internalization of the receptor in cancer cells and degradation, ultimately resulting in the inhibition of cancer-cell growth. In vitro and in vivo evidence demonstrates that Sym004 can be a superior agent if compared to both cetuximab and panitumumab in a wide range of tumor types, with a clear dose-response relationship. ${ }^{149}$ Furthermore, Sym004 inhibits growth and proliferation of those cancer cells that have acquired resistance to anti-EGFR therapies. This acquired resistance represents a common dilemma in patients treated with anti-EGFR therapeutic agents, leaving numerous mechanisms by which tumors are capable of escaping inhibition, including increased EGFR ligand production surrounding the tumor. Sym004 potentially inhibits proliferation also in the presence of increased EGF concentrations.

The first-in-human trial did not show unexpected toxicities, and based on preliminary signs of clinical activity, Sym004 has been tested as monotherapy in selected patients with KRAS wt CRC progressing to previous cetuximab- or panitumumab-based therapy. ${ }^{150}$ In total, 42 patients were enrolled. Tumor shrinkage $>10 \%$ was documented in four of $12(33 \%)$ patients at $9 \mathrm{mg} / \mathrm{kg}$, with partial response in one of $12(8 \%)$ and stable disease in nine of $12(75 \%)$. At $12 \mathrm{mg} / \mathrm{kg}$, seven of 27 (26\%) patients had $>10 \%$ tumor shrinkage, with partial response in three of $27(11 \%)$ and stable disease in 15 of 27 (56\%). Median PFS was 13.6 weeks (95\% confidence interval 5.3-23) and 13.7 weeks (95\% confidence interval 5.9-18.6), respectively. Sym004 showed significant clinical activity in anti-EGFR treatment-refractory $K R A S$ wt mCRC patients, and serial biopsies confirmed its mechanism of action. No unexpected adverse events were observed. The agent is currently being tested as monotherapy in a Phase II trial, compared to investigator's choice (BSC or 5-FU or capecitabine) in subjects with $\mathrm{mCRC}$ and acquired resistance to anti-EGFR mAbs (NCT02083653). ${ }^{151}$

\section{Anti-EGFR in combination with mAbs directed to other receptors}

The extensive cross talk among the HER-family receptors is most likely responsible for emerging reports that blockade of a particular signaling pathway can lead to compensatory actions, such as negative-feedback loops and consequently to the upregulation of parallel pathways.

One potential mechanism of resistance to anti-EGFR therapy is related to the ability of EGFR to form heterodimers with HER3, producing receptor autophosphorylation

Table 2 Selected combinations of anti-EGFR mAbs and other targeted agents in colorectal cancer

\begin{tabular}{|c|c|c|c|c|c|c|}
\hline EGFR inhibitor & Agent & Class & Target & Phase & Results & Study/trial \\
\hline \multirow[t]{6}{*}{ Cetuximab } & IMC-AI2 & $\mathrm{mAb}$ & IGF-IR & II & Negative & Reidy et al ${ }^{159}$ \\
\hline & Dalotuzumab & & & II & Negative (worsened survival) & Watkins et al ${ }^{160}$ \\
\hline & BMS-754807 & TKI & IGF-IR & $|-I|$ & Completed & NCT00908024166 \\
\hline & ARQ 197 & TKI & c-MET & $\mathrm{IB} / \mathrm{II}$ & $\begin{array}{l}\text { Promising (increase in OS, } \\
\text { not significant) }\end{array}$ & Eng et al ${ }^{161}$ \\
\hline & LY280I653 & & & 1 & Ongoing & NCT0I $285037^{167}$ \\
\hline & EMD525797 & $\mathrm{mAb}$ & $\alpha$-Integrin & II & Ongoing & NCTOI008475 168 \\
\hline \multirow[t]{3}{*}{ Panitumumab } & AMG 479 & $\mathrm{mAb}$ & IGF-IR & $\mathrm{I} / \mathrm{II}$ & Negative & Van Cutsem et al ${ }^{162}$ \\
\hline & AMG 102 & & HGF/SF & & Promising (increase in RR) & \\
\hline & AMG 655 & & TRAIL-R2 & $\mid \mathrm{IB} / \mathrm{II}$ & Negative & Peeters et $\mathrm{al}^{163}$ \\
\hline
\end{tabular}

Abbreviations: $\mathrm{mAb}$, monoclonal antibody; OS, overall survival; RR, response rate; TKI, tyrosine-kinase inhibitor; EGFR, epidermal growth-factor receptor. 
Table 3 Selected combination trials of anti-EGFR monoclonal antibodies and other agents targeting downstream signaling pathways in colorectal cancer

\begin{tabular}{|c|c|c|c|c|c|}
\hline EGFR inhibitor & Agent & Pathway & Phase & Results & Trial/study \\
\hline \multirow[t]{9}{*}{ Cetuximab } & Sorafenib & $\begin{array}{l}\text { VEGFR, PDGFR, RAF, } \\
\text { FLT-3, c-KIT, RET }\end{array}$ & II & Ongoing & NCT00326495 169 \\
\hline & Ridaforolimus & mTOR & IB & Negative & Taber et $\mathrm{al}^{164}$ \\
\hline & Tensirolimus & & IB & Completed & NCT00593060170 \\
\hline & Everolimus & & $\mathrm{IB} / \mathrm{II}$ & Ongoing & NCT00522665 \\
\hline & PX-866 & PI3K & II & Ongoing & NCTOI $252628^{172}$ \\
\hline & PF-052I2384 & $\mathrm{PI} 3 \mathrm{~K} / \mathrm{mTOR}$ & II & Ongoing & NCTOI925274 173 \\
\hline & AZD6244 & MEK & IB & Promising & Deming et $\mathrm{al}^{165}$ \\
\hline & LGX8I8, BYL7I9 & BRAF, PI3K & $\mathrm{IB} / \mathrm{II}$ & Ongoing & NCTOI7I9380174 \\
\hline & Vemurafenib & BRAF & IB & Ongoing & NCTOI524978 175 \\
\hline \multirow[t]{2}{*}{ Panitumumab } & Dabrafenib/trametinib & BRAF/MEK & II & Ongoing & NCT017509/8176 \\
\hline & MEKI 62 & MEK & $\mathrm{IB} / \mathrm{II}$ & Ongoing & NCTOI92734|177 \\
\hline
\end{tabular}

Abbreviation: EGFR, epidermal growth-factor receptor.

and leading to intracellular signaling activation, mainly via the PI3K-AKT-mTOR pathway. In the clinical setting, HER3 has been associated with tumor resistance to therapeutic agents targeting EGFR or HER2 in lung and breast cancer, ${ }^{152}$ and its expression correlates with a poor outcome in mCRC patients treated with cetuximab. ${ }^{153}$ These findings led to the hypothesis that inhibiting the signaling of more than one of the HER-family receptors offers an opportunity for greater efficiency and the potential for overcoming resistance to currently available EGFR-directed therapies. As a result, MEHD7945A, an $\mathrm{IgG}_{1} \mathrm{mAb}$, was developed to bind simultaneously with high affinity to EGFR and HER3. As an $\mathrm{IgG}_{1}$ antibody, MEHD7945A is also able to bind to Fcy receptors, has the potential to elicit antibody-dependent cellular cytotoxicity, and has also been demonstrated to have significant activity in colon, lung, pancreatic, head and neck, breast, and ovarian xenograft models, exhibiting broader activity compared to other monospecific HER family-targeting agents. The Phase I first-in-human trial, with an expansion at the recommended dose in KRAS wt CRC patients, showed an encouraging safety profile and evidence of antitumor activity. ${ }^{154}$

Similarly, HER 2 is amplified only in $2 \%-3 \%$ of genetically unselected $\mathrm{mCRC}$, but its increased overexpression has been associated with both de novo and acquired resistance to cetuximab-based therapy in CRC patients, and in the subset of KRAS/NRAS/BRAF/PIK3CA wt "xenopatients" resistant to cetuximab, HER2 amplification was observed in up to $36 \%$. ${ }^{155-157}$

Lastly, as a proof of concept, the inhibition of EGFR and HER2 was shown to induce overt, long-lasting tumor regression in HER2-amplified xenopatients. Based on this rationale, an ongoing Phase IB/II trial is exploring the combination of neratinib, an HER2 TKI, with cetuximab, in patients with "quadruple wt" (wt KRAS, NRAS, BRAF, PIK3CA) mCRC primary tumor (NCT01960023). ${ }^{158}$

Combination strategies with other membrane-bound receptors, such as c-MET and IGFR, have been translated in completed or ongoing Phase I/II trials. Thus far, none have proven very effective clinically (Table 2). ${ }^{159-163}$

\section{Downstream effector inhibitors}

The most promising approaches to circumvent or reverse resistance to anti-EGFR-targeted therapies are rational combinations of targeted treatments that include inhibitors of downstream effectors of the EGFR pathway. Currently, several drugs capable of inhibiting activated BRAF, MEK, PI3K, AKT, and mTOR are available. Furthermore, clinical trials with these agents are actively recruiting patients, and for some of these trials, the selection of therapy is based on the genetic profile of the tumor, as shown in Table 3.

\section{Conclusion}

The EGFR signaling pathway plays a pivotal role in CRC progression and treatment. Therefore, targeted therapies (mAbs) against this marker have been developed and have entered clinical practice. A myriad of studies focused attention on the possibility of predicting the efficacy of EGFR-targeted therapies, and currently the assessment of KRAS and NRAS gene mutations is mandatory before any administration of anti-EGFR mAbs. However, it has also been demonstrated that the molecular characterization of the EGFR pathway, due to technical problems as well as by the co-occurrence of different genetic alterations in the same patient (rendering it difficult to understand the clinical role of the individual 
alteration), needs to be refined. Therefore, the efforts of molecular pathologists are currently addressed toward investigating the aforementioned problem.

On the other hand, pharmaceutical companies have studied new modalities of the administration of cetuximab and panitumumab, as well as developing new (and hypothetically more efficient) compounds, not only targeted against EGFR, but also against EGFR-downstream members (to be administered in combination with anti-EGFR mAbs). These new options are the object of intensive studies, and may also lead to a substantial improvement for patients affected by an EGFR-addicted CRC.

Given all these considerations, there is reasonable hope that mCRC patients will be better treated in the near future.

\section{Disclosure}

The authors report no conflicts of interest in this work.

\section{References}

1. Jemal A, Center MM, DeSantis C, Ward EM. Review Global patterns of cancer incidence and mortality rates and trends. Cancer Epidemiol Biomarkers Prev. 2010;19:1893-1907.

2. International Agency for Research on Cancer. Globocan 2008 cancer fact sheet. 2010. Available from: http://globocan.iarc.fr/Pages/ fact_sheets_cancer.aspx. Accessed November 11, 2014.

3. Schmoll HJ, Van Cutsem E, Stein A, et al. ESMO Consensus Guidelines for management of patients with colon and rectal cancer. A personalized approach to clinical decision making. Ann Oncol. 2012;23:2479-2516.

4. Stintzing S, Fischer von Weikersthal L, Decker T, et al. FOLFIRI plus cetuximab versus FOLFIRI plus bevacizumab as first-line treatment for patients with metastatic colorectal cancer-subgroup analysis of patients with KRAS: mutated tumours in the randomised German AIO study KRK-0306. Ann Oncol. 2012;23:1693-1699.

5. Van Cutsem E, Köhne CH, Láng I, et al. Cetuximab plus irinotecan, fluorouracil, and leucovorin as first-line treatment for metastatic colorectal cancer: updated analysis of overall survival according to tumor KRAS and BRAF mutation status. J Clin Oncol. 2011;29:2011-2019.

6. Douillard JY, Siena S, Cassidy J, et al. Randomized, phase III trial of panitumumab with infusional fluorouracil, leucovorin, and oxaliplatin (FOLFOX4) versus FOLFOX4 alone as first-line treatment in patients with previously untreated metastatic colorectal cancer: the PRIME study. J Clin Oncol. 2010;28:4697-4705.

7. Kopetz S, Chang GJ, Overman MJ, et al. Improved survival in metastatic colorectal cancer is associated with adoption of hepatic resection and improved chemotherapy. J Clin Oncol. 2009;27:3677-3683.

8. Falcone A, Cremolini C, Masi G, et al. FOLFOXIRI/bevacizumab (bev) versus FOLFIRI/bev as first-line treatment in unresectable metastatic colorectal cancer (mCRC) patients (pts): results of the phase III TRIBE trial by GONO group. J Clin Oncol. 2013;Suppl 31:3505.

9. Lise M, Da Pian PP, Nitti D, Pilati PL. Colorectal metastases to the liver: present results and future strategies. J Surg Oncol Suppl. 1991;2:69-73.

10. Bismuth H, Adam R, Lévi F, et al. Resection of nonresectable liver metastases from colorectal cancer after neoadjuvant chemotherapy. Ann Surg. 1996;224:509-520.

11. Folprecht G, Gruenberger T, Bechstein W, et al. Survival of patients with initially unresectable colorectal liver metastases treated with FOLFOX/cetuximab or FOLFIRI/cetuximab in a multidisciplinary concept (CELIM study). Ann Oncol. 2014;25:1018-1025.
12. Ruers T, Punt C, Van Coevorden F, et al. Radiofrequency ablation combined with systemic treatment versus systemic treatment alone in patients with non-resectable colorectal liver metastases: a randomized EORTC Intergroup phase II study (EORTC 40004). Ann Oncol. 2012;23:2619-2626.

13. Tournigand C, André T, Achille E, et al. FOLFIRI followed by FOLFOX6 or the reverse sequence in advanced colorectal cancer: a randomized GERCOR study. J Clin Oncol. 2004;22:229-237.

14. Seymour MT, Maughan TS, Ledermann JA, et al. Different strategies of sequential and combination chemotherapy for patients with poor prognosis advanced colorectal cancer (MRC FOCUS): a randomised controlled trial. Lancet. 2007;370:143-152.

15. Falcone A, Ricci S, Brunetti I, et al. Phase III trial of infusional fluorouracil, leucovorin, oxaliplatin, and irinotecan (FOLFOXIRI) compared with infusional fluorouracil, leucovorin, and irinotecan (FOLFIRI) as first-line treatment for metastatic colorectal cancer: the Gruppo Oncologico Nord Ovest. J Clin Oncol. 2007;25:1670-1676.

16. Koopman M, Antonini NF, Douma J, et al. Sequential versus combination chemotherapy with capecitabine, irinotecan, and oxaliplatin in advanced colorectal cancer (CAIRO): a phase III randomised controlled trial. Lancet. 2007;370:135-142.

17. Ducreux M, Malka D, Mendiboure J, et al. Sequential versus combination chemotherapy for the treatment of advanced colorectal cancer (FFCD 2000-05): an open-label, randomised, phase 3 trial. Lancet Oncol. 2011;12:1032-1044.

18. Tournigand C, Cervantes A, Figer A, et al. OPTIMOX1: a randomized study of FOLFOX4 or FOLFOX7 with oxaliplatin in a stop-and-go fashion in advanced colorectal cancer - a GERCOR study. J Clin Oncol. 2006;24:394-400.

19. Chibaudel B, Maindrault-Goebel F, Lledo G, et al. Can chemotherapy be discontinued in unresectable metastatic colorectal cancer? The GERCOR OPTIMOX2 Study. J Clin Oncol. 2009;27: 5727-5733.

20. Koopman M, Simkens LH, Ten Tije AJ, et al. Maintenance treatment with capecitabine and bevacizumab versus observation after induction treatment with chemotherapy and bevacizumab in metastatic colorectal cancer (mCRC): the phase III CAIRO3 study of the Dutch Colorectal Cancer Group (DCCG). J Clin Oncol. 2013;Suppl 31:3502.

21. Price TJ, Townsend AR, Beeke C, et al. "Watchful waiting" for metastatic colorectal cancer, antediluvian or an option to be considered again? Asia Pac J Clin Oncol. 2012;8:10-13.

22. Kabbinavar FF, Hambleton J, Mass RD, et al. Combined analysis of efficacy: the addition of bevacizumab to fluorouracil/leucovorin improves survival for patients with metastatic colorectal cancer. J Clin Oncol. 2005;23:3706-3712.

23. Hurwitz H, Fehrenbacher L, Novotny W, et al. Bevacizumab plus irinotecan, fluorouracil, and leucovorin for metastatic colorectal cancer. N Engl J Med. 2004;350:2335-2342.

24. Saltz LB, Clarke S, Díaz-Rubio E, et al. Bevacizumab in combination with oxaliplatin-based chemotherapy as first-line therapy in metastatic colorectal cancer: a randomized phase III study. J Clin Oncol. 2008;26:2013-2019.

25. Giantonio BJ, Catalano PJ, Meropol NJ, et al. Bevacizumab in combination with oxaliplatin, fluorouracil, and leucovorin (FOLFOX4) for previously treated metastatic colorectal cancer: results from the Eastern Cooperative Oncology Group Study E3200. J Clin Oncol. 2007;25:1539-1544.

26. Kubicka S, Greil R, André T, et al. Bevacizumab plus chemotherapy continued beyond first progression in patients with metastatic colorectal cancer previously treated with bevacizumab plus chemotherapy: ML18147 study KRAS subgroup findings. Ann Oncol. 2013;24:2342-2349.

27. Cunningham D, Lang I, Marcuello E, et al. Bevacizumab plus capecitabine versus capecitabine alone in elderly patients with previously untreated metastatic colorectal cancer (AVEX): an open-label, randomised phase 3 trial. Lancet Oncol. 2013;14:1077-1085. 
28. Van Cutsem E, Tabernero J, Lakomy R, et al. Addition of aflibercept to fluorouracil, leucovorin, and irinotecan improves survival in a phase III randomized trial in patients with metastatic colorectal cancer previously treated with an oxaliplatin-based regimen. J Clin Oncol. 2012;30:3499-3506.

29. Grothey A, Van Cutsem E, Sobrero A, et al. Regorafenib monotherapy for previously treated metastatic colorectal cancer (CORRECT): an international, multicentre, randomised, placebo-controlled, phase 3 trial. Lancet. 2013;381:303-312.

30. Mitsudomi T, Yatabe Y. Epidermal growth factor receptor in relation to tumor development: EGFR gene and cancer. FEBS J. 2010;277: 301-308.

31. Woodburn JR. The epidermal growth factor receptor and its inhibition in cancer therapy. Pharmacol Ther. 1999;82:241-250.

32. Arteaga CL. The epidermal growth factor receptor: from mutant oncogene in nonhuman cancers to therapeutic target in human neoplasia. J Clin Oncol 2001;Suppl 18:32S-40S.

33. Talapatra S, Thompson CB. Growth factor signalling in cell survival: implications for cancer treatment. J Pharmacol Exp Ther. 2004;298:873-878.

34. Venook A. Critical evaluation of current treatments in metastatic colorectal cancer. Oncologist. 2005;10:250-261.

35. Custodio A, Feliu J. Prognostic and predictive biomarkers for epidermal growth factor receptor-targeted therapy in colorectal cancer: beyond KRAS mutations. Crit Rev Oncol Hematol. 2013;85:45-81.

36. Anastasi S, Fiorentino L, Fiorini M, et al. Feedback inhibition by RALT controls signal output by the ErbB network. Oncogene. 2003;22:4221-4234.

37. Gur G, Rubin C, Katz M, et al. LRIG1 restricts growth factor signaling by enhancing receptor ubiquitylation and degradation. EMBO J. 2004;23:3270-3281.

38. Laederich MB, Funes-Duran M, Yen L, et al. The leucine-rich repeat protein LRIG1 is a negative regulator of ErbB family receptor tyrosine kinases. J Biol Chem. 2004;279:47050-47056.

39. Marmor MD, Yarden Y. Role of protein ubiquitylation in regulating endocytosis of receptor tyrosine kinases. Oncogene. 2004;23:2057-2070.

40. Kario E, Marmor MD, Adamsky K, et al. Suppressors of cytokine signaling 4 and 5 regulate epidermal growth factor receptor signaling. J Biol Chem. 2005;280:7038-7048.

41. Tarcic G, Boguslavsky SK, Wakim J, et al. An unbiased screen identifies DEP-1 tumor suppressor as a phosphatase controlling EGFR endocytosis. Curr Biol. 2009;19:1788-1798.

42. Mc Kay JA, Murray LJ, Curran S, et al. Evaluation of the epidermal growth factor receptor (EGFR) in colorectal tumors and lymph node metastases. Eur J Cancer. 2002;38:2258-2264.

43. Mendelsohn J, Baselga J. Epidermal growth factor receptor targeting in cancer. Semin Oncol. 2006;33:369-385.

44. Paez JG, Jänne PA, Lee JC, et al. EGFR mutations in lung cancer: correlation with clinical response to gefitinib therapy. Science. 2004;304:1497-1500.

45. Pao W, Miller V, Zakowski M, et al. EGF receptor gene mutations are common in lung cancers from "never smokers" and are associated with sensitivity of tumors to gefitinib and erlotinib. Proc Natl Acad Sci US A. 2004;101:13306-13311.

46. Ogino S, Meyerhardt JA, Cantor M, et al. Molecular alterations in tumors and response to combination chemotherapy with gefitinib for advanced colorectal cancer. Clin Cancer Res. 2005;11:6650-6656.

47. Bonomi PD, Buckingham L, Coon J. Selecting patients for treatment with epidermal growth factor tyrosine kinase inhibitors. Clin Cancer Res. 2007;13:s4606-s4612.

48. Yarom N, Jonker DJ. The role of the epidermal growth factor receptor in the mechanism and treatment of colorectal cancer. Discov Med. 2011;11:95-105.

49. Goldstein NS, Armin M. Epidermal growth factor receptor immunohistochemical reactivity in patients with American Joint Committee on Cancer Stage IV colon adenocarcinoma: implications for a standardized scoring system. Cancer. 2001;92:1331-1346.
50. Resnick MB, Routhier J, Konkin T, Sabo E, Pricolo VE. Epidermal growth factor receptor, c-MET, beta-catenin, and p53 expression as prognostic indicators in stage II colon cancer: a tissue microarray study. Clin Cancer Res. 2004;10:3069-3075.

51. Galizia G, Lieto E, Ferraraccio F, et al. Prognostic significance of epidermal growth factor receptor expression in colon cancer patients undergoing curative surgery. Ann Surg Oncol. 2006;13:823-835.

52. Ljuslinder I, Melin B, Henriksson ML, Öberg Å, Palmqvist R. Increased epidermal growth factor receptor expression at the invasive margin is a negative prognostic factor in colorectal cancer. Int $J$ Cancer. 2011;128:2031-2037.

53. Rego RL, Foster NR, Smyrk TC, et al. Prognostic effect of activated EGFR expression in human colon carcinomas: comparison with EGFR status. Br J Cancer. 2010;102:165-172.

54. Lee JC, Wang ST, Chow NH, Yang HB. Investigation of the prognostic value of coexpressed erbB family members for the survival of colorectal cancer patients after curative surgery. Eur J Cancer. 2002;38:1065-1071.

55. Spano JP, Lagorce C, Atlan D, et al. Impact of EGFR expression on colorectal cancer patient prognosis and survival. Ann Oncol. 2005; 16:102-108

56. Leung SP, Griffith OL, Masoudi H, et al. Clinical utility of type 1 growth factor receptor expression in colon cancer. Am J Surg. 2008;195:604-610.

57. Grünwald V, Hidalgo M. Developing inhibitors of the epidermal growth factor receptor for cancer treatment. $J$ Natl Cancer Inst. 2003;95:851-867.

58. Ciardiello F, Tortora G. EGFR antagonists in cancer treatment. $N$ Engl JMed. 2008;358:1160-1174.

59. Jonker DJ, O'Callaghan CJ, Karapetis CS, et al. Cetuximab for the treatment of colorectal cancer. N Engl J Med. 2007;357:2040-2048.

60. Amado RG, Wolf M, Peeters M, et al. Wild-type KRAS is required for panitumumab efficacy in patients with metastatic colorectal cancer. J Clin Oncol. 2008;26:1626-1634.

61. Kimura H, Sakai K, Arao T, Shimoyama T, Tamura T, Nishio K. Antibody-dependent cellular cytotoxicity of cetuximab against tumor cells with wild-type or mutant epidermal growth factor receptor. Cancer Sci 2007;98:1275-1280.

62. Kurai J, Chikumi H, Hashimoto K, et al. Antibody dependent cellular cytotoxicity mediated by cetuximab against lung cancer cell lines. Clin Cancer Res. 2007;13:1552-1561.

63. Baselga J, Pfister D, Cooper MR, et al. Phase I studies of antiepidermal growth factor receptor chimeric antibody $\mathrm{C} 225$ alone and in combination with cisplatin. J Clin Oncol. 2000;18:904-914.

64. Prewett MC, Hooper AT, Bassi R, et al. Enhanced antitumor activity of anti-epidermal growth factor receptor monoclonal antibody IMC-C225 in combination with irinotecan (CPT-11) against human colorectal tumor xenografts. Clin Cancer Res. 2002;8:994-1003.

65. Cunningham D, Humblet Y, Siena S, et al. Cetuximab monotherapy and cetuximab plus irinotecan in irinotecan-refractory metastatic colorectal cancer. $N$ Engl J Med. 2004:351:337-345.

66. Saltz LB, Meropol NJ, Loehrer PJ Sr, et al. Phase II trial of cetuximab in patients with refractory colorectal cancer that expresses the epidermal growth factor receptor. J Clin Oncol. 2004;22:1201-1208.

67. Chung KY, Shia J, Kemeny NE, et al. Cetuximab shows activity in colorectal cancer patients with tumors that do not express the epidermal growth factor receptor by immunohistochemistry. J Clin Oncol. 2005;23:1803-1810.

68. Pérez-Soler R, Chachoua A, Hammond LA, et al. Determinants of tumor response and survival with erlotinib in patients with non-small-cell lung cancer. J Clin Oncol. 2004;22:3238-3247.

69. Tol J, Koopman M, Cats A, et al. Chemotherapy, bevacizumab and cetuximab in metastatic colorectal cancer. N Engl J Med. 2009;360:563-572.

70. Maughan TS, Adams RA, Smith CG, et al. Addition of cetuximab to oxaliplatin-based first-line combination chemotherapy for treatment of advanced colorectal cancer: results of the randomised phase 3MRC COIN trial. Lancet. 2011;377:2103-2114. 
71. Atkins D, Reiffen KA, Tegtmeier CL, Winther H, Bonato MS, Störkel S. Immunohistochemical detection of EGFR in paraffin-embedded tumor tissues: variation in staining intensity due to choice of fixative and storage time of tissue sections. $J$ Histochem Cytochem. 2004;52:893-901.

72. Langner C, Ratschek M, Rehak P, Schips L, Zigeuner R. Are heterogenous results of EGFR immunoreactivity in renal cell carcinoma related to non-standardised criteria for staining evaluation? J Clin Pathol. 2004;57:773-775.

73. Kersting C, Packeisen J, Leidinger B, et al. Pitfalls in immunohistochemical assessment of EGFR expression in soft tissue sarcomas. J Clin Pathol. 2006;59:585-590.

74. Shia J, Klimstra DS, Li AR, et al. Epidermal growth factor receptor expression and gene amplification in colorectal carcinoma: an immunohistochemical and chromogenic in situ hybridization study. Mod Pathol. 2005;18:1350-1356.

75. Cappuzzo F, Finocchiaro G, Rossi E, et al. EGFR FISH assay predicts for response to cetuximab in chemotherapy refractory colorectal cancer patients. Ann Oncol. 2008;19:717-723.

76. Bardelli A, Siena S. Molecular mechanisms of resistance to cetuximab and panitumumab in colorectal cancer. J Clin Oncol. 2010;28:1254-1261

77. Moroni M, Veronese S, Benvenuti S, et al. Gene copy number for epidermal growth factor receptor (EGFR) and clinical response to antiEGFR treatment in colorectal cancer: a cohort study. Lancet Oncol. 2005;6:279-286.

78. Lièvre A, Bachet JB, Le Corre D, et al. KRAS mutation status is predictive of response to cetuximab therapy in colorectal cancer. Cancer Res. 2006;66:3992-3995.

79. Frattini M, Saletti P, Romagnani E, et al. PTEN loss of expression predicts cetuximab efficacy in metastatic colorectal cancer patients. Br J Cancer. 2007;97:1139-1145.

80. Sartore-Bianchi A, Moroni M, Veronese S, et al: Epidermal growth factor receptor gene copy number and clinical outcome of metastatic colorectal cancer treated with panitumumab. J Clin Oncol. 2007;25:3238-3245.

81. Personeni N, Fieuws S, Piessevaux H, et al. Clinical usefulness of EGFR gene copy number as a predictive marker in colorectal cancer patients treated with cetuximab: a fluorescent in situ hybridization study. Clin Cancer Res. 2008;14:5869-5876.

82. Italiano A, Follana P, Caroli FX, et al. Cetuximab shows activity in colorectal cancer patients with tumors for which FISH analysis does not detect an increase in EGFR gene copy number. Ann Surg Oncol. 2008;15:649-654.

83. Laurent-Puig P, Cayre A, Manceau G, et al. Analysis of PTEN, BRAF, and EGFR status in determining benefit from cetuximab therapy in wild-type KRAS metastatic colon cancer. J Clin Oncol. 2009;27:5924-5930

84. Scartozzi M, Bearzi I, Mandolesi A, et al. Epidermal growth factor receptor (EGFR) gene copy number $(\mathrm{GCN})$ correlates with clinical activity of irinotecan-cetuximab in K-RAS wild-type colorectal cancer: a fluorescence in situ (FISH) and chromogenic in situ hybridization (CISH) analysis. BMC Cancer. 2009;9:303.

85. Martin V, Mazzucchelli L, Frattini M. An overview of the epidermal growth factor receptor fluorescence in situ hybridisation challenge in tumour pathology. J Clin Pathol. 2009;62:314-324.

86. Sartore-Bianchi A, Fieuws S, Veronese S, et al. Standardisation of EGFR FISH in colorectal cancer: results of an international interlaboratory reproducibility ring study. J Clin Pathol. 2012;65:218-223.

87. Khambata-Ford S, Garrett CR, Meropol NJ, et al. Expression of epiregulin and amphiregulin and K-ras mutation status predict disease control in metastatic colorectal cancer patients treated with cetuximab. J Clin Oncol. 2007;25:3230-3237.

88. Jacobs B, De Roock W, Piessevaux H, et al. Amphiregulin and epiregulin mRNA expression in primary tumors predicts outcome in metastatic colorectal cancer treated with cetuximab. J Clin Oncol. 2009;27:5068-5074.
89. Baker JB, Dutta D, Watson D, et al. Tumour gene expression predicts response to cetuximab in patients with KRAS wild-type metastatic colorectal cancer. Br J Cancer. 2011;104:488-495.

90. Saridaki Z, Tzardi M, Papadaki C, et al. Impact of KRAS, BRAF, PIK3CA mutations, PTEN, AREG, EREG expression and skin rash in $\geq 2$ line cetuximab-based therapy of colorectal cancer patients. PLoS One. 2011;6:e15980.

91. Jonker DJ, Karapetis CS, Harbison C, et al. Epiregulin gene expression as a biomarker of benefit from cetuximab in the treatment of advanced colorectal cancer. Br J Cancer. 2014;110:648-655.

92. De Roock W, Jonker DJ, Di Nicolantonio F, et al. Association of KRAS p.G13D mutation with outcome in patients with chemotherapyrefractory metastatic colorectal cancer treated with cetuximab. JAMA. 2010;304:1812-1820.

93. Edkins S, O'Meara S, Parker A, et al. Recurrent KRAS codon 146 mutations in human colorectal cancer. Cancer Biol Ther. 2006;5:928-932.

94. Kosaka T, Yatabe Y, Endoh H, et al. Mutations of the epidermal growth factor receptor gene in lung cancer: biological and clinical implications. Cancer Res. 2004;64:8919-8923.

95. Douillard JY, Oliner KS, Siena S, et al. Panitumumab-FOLFOX4 treatment and RAS mutations in colorectal cancer. $N$ Engl $J$ Med. 2013;369:1023-1034.

96. Peeters M, Oliner KS, Parker A, et al. Massively parallel tumor multigene sequencing to evaluate response to panitumumab in a randomized phase III study of metastatic colorectal cancer. Clin Cancer Res. 2013;19:1902-1912.

97. Loupakis F, Ruzzo A, Cremolini C, et al. KRAS codon 61, 146 and BRAF mutations predict resistance to cetuximab plus irinotecan in KRAS codon 12 and 13 wild-type metastatic colorectal cancer. $\mathrm{Br} J$ Cancer. 2009;101:715-721.

98. Michaloglou C, Vredeveld LC, Mooi WJ, Peeper DS. BRAF(E600) in benign and malignant human tumours. Oncogene. 2008;27: 877-895.

99. Rajagopalan H, Bardelli A, Lengauer C, Kinzler KW, Vogelstein B, Velculescu VE. Tumorigenesis: RAF/RAS oncogenes and mismatchrepair status. Nature. 2002;418:934.

100. Davies H, Bignell GR, Cox C, et al. Mutations of the BRAF gene in human cancer. Nature. 2002;417:949-954.

101. Di Nicolantonio F, Martini M, Molinari F, et al. Wild-type BRAF is required for response to panitumumab or cetuximab in metastatic colorectal cancer. J Clin Oncol. 2008;26:5705-5712.

102. Seymour MT, Brown SR, Middleton G, et al. Panitumumab and irinotecan versus irinotecan alone for patients with KRAS wild-type, fluorouracil-resistant advanced colorectal cancer (PICCOLO): a prospectively stratified randomised trial. Lancet Oncol. 2013;14:749-759.

103. Bokemeyer C, Van Cutsem E, Rougier P, et al. Addition of cetuximab to chemotherapy as first-line treatment for KRAS wild-type metastatic colorectal cancer: pooled analysis of the CRYSTAL and OPUS randomised clinical trials. Eur J Cancer. 2012;48:1466-1475.

104. Yuan TL, Cantley LC. PI3K pathway alterations in cancer: variations on a theme. Oncogene. 2008;27:5497-5510.

105. Jhawer M, Goel S, Wilson AJ, et al. PIK3CA mutation/PTEN expression status predicts response of colon cancer cells to the epidermal growth factor receptor inhibitor cetuximab. Cancer Res. 2008;68:1953-1961.

106. Sartore-Bianchi A, Martini M, Molinari F, et al. PIK3CA mutations in colorectal cancer are associated with clinical resistance to EGFR-targeted monoclonal antibodies. Cancer Res. 2009;69: 1851-1857.

107. Perrone F, Lampis A, Orsenigo M, et al. PI3KCA/PTEN deregulation contributes to impaired responses to cetuximab in metastatic colorectal cancer patients. Ann Oncol. 2009;20:84-90.

108. Prenen H, De Schutter J, Jacobs B, et al. PIK3CA mutations are not a major determinant of resistance to the epidermal growth factor receptor inhibitor cetuximab in metastatic colorectal cancer. Clin Cancer Res. 2009;15:3184-3188. 
109. Sansal I, Sellers WR. The biology and clinical relevance of the PTEN tumor suppressor pathway. J Clin Oncol. 2004;22:2954-2963.

110. Razis E, Briasoulis E, Vrettou E, et al. Potential value of PTEN in predicting cetuximab response in colorectal cancer: an exploratory study. BMC Cancer. 2008;8:234.

111. Sartore-Bianchi A, Di Nicolantonio F, Nichelatti M, et al. Multi-determinants analysis of molecular alterations for predicting clinical benefit to EGFR-targeted monoclonal antibodies in colorectal cancer. PLoS One. 2009;4:e7287.

112. Ulivi P, Capelli L, Valgiusti M, et al. Predictive role of multiple gene alterations in response to cetuximab in metastatic colorectal cancer: a single center study. J Transl Med. 2012;10:87.

113. Van Cutsem E, Köhne CH, Hitre E, et al. Cetuximab and chemotherapy as initial treatment for metastatic colorectal cancer. $N$ Engl $J$ Med. 2009;360:1408-1417.

114. Bokemeyer C, Bondarenko I, Makhson A, et al. Fluorouracil, leucovorin, and oxaliplatin with and without cetuximab in the first-line treatment of metastatic colorectal cancer. J Clin Oncol. 2009;27:663-671.

115. Bokemeyer C, Bondarenko I, Hartmann JT, et al. Efficacy according to biomarker status of cetuximab plus FOLFOX-4 as first-line treatment for metastatic colorectal cancer: the OPUS study. Ann Oncol. 2011;22:1535-1546.

116. Tveit KM, Guren T, Glimelius B, et al. Phase III trial of cetuximab with continuous or intermittent fluorouracil, leucovorin, and oxaliplatin (Nordic FLOX) versus FLOX alone in first-line treatment of metastatic colorectal cancer: the NORDIC-VII study. J Clin Oncol. 2012;30:1755-1762.

117. Heinemann V, Fischer von Weikersthal L, Decker T, et al. Randomized comparison of FOLFIRI plus cetuximab versus FOLFIRI plus bevacizumab as first-line treatment of KRAS wild-type metastatic colorectal cancer: German AIO study KRK-0306 (FIRE-3). J Clin Oncol. 2013;31 Suppl 18:LBA3506.

118. Stintzing S, Jung A, Rossius L. Analysis of KRAS/NRAS and BRAF mutations in FIRE-3: a randomized phase III study of FOLFIRI plus cetuximab or bevacizumab as first-line treatment for wild-type (WT) KRAS (exon 2) metastatic colorectal cancer (mCRC) patients. Poster presented at: European Cancer Congress 2013; September $27-$ October 1, 2013; Amsterdam, The Netherlands.

119. Schwartzberg LS, Rivera F, Karthaus M, et al. PEAK: a randomized, multicenter phase II study of panitumumab plus modified fluorouracil, leucovorin, and oxaliplatin (mFOLFOX6) or bevacizumab plus mFOLFOX6 in patients with previously untreated, unresectable, wild-type KRAS exon 2 metastatic colorectal cancer. J Clin Oncol. 2014;32:2240-2247.

120. Venook AP, Niedzwiecki D, HJ et al. CALGB/SWOG 80405: phase III trial of irinotecan/5-FU/leucovorin (FOLFIRI) or oxaliplatin/5-FU/ leucovorin (mFOLFOX6) with bevacizumab (BV) or cetuximab (CET) for patients (pts) with KRAS wild-type (wt) untreated metastatic adenocarcinoma of the colon or rectum (MCRC). J Clin Oncol. 2014;Suppl 32:LBA3.

121. Giessen C, Laubender RP, Fischer von Weikersthal L, et al. Early tumor shrinkage in metastatic colorectal cancer: retrospective analysis from an irinotecan-based randomized first-line trial. Cancer Sci. 2013;104:718-724.

122. Modest DP, Laubender RP, Stintzing S, et al. Early tumor shrinkage in patients with metastatic colorectal cancer receiving first-line treatment with cetuximab combined with either CAPIRI or CAPOX: an analysis of the German AIO KRK 0104 trial. Acta Oncol. 2013;52:956-962.

123. Folprecht G, Gruenberger T, Bechstein WO, et al. Tumour response and secondary resectability of colorectal liver metastases following neoadjuvant chemotherapy with cetuximab: the CELIM randomised phase 2 trial. Lancet Oncol. 2010;11:38-47.

124. Ye LC, Liu TS, Ren L, et al. Randomized controlled trial of cetuximab plus chemotherapy for patients with KRAS wild-type unresectable colorectal liver-limited metastases. J Clin Oncol. 2013;31: 1931-1938.
125. Saridaki Z, Androulakis N, Vardakis N, et al. A triplet combination with irinotecan (CPT-11), oxaliplatin (LOHP), continuous infusion 5 -fluorouracil and leucovorin (FOLFOXIRI) plus cetuximab as firstline treatment in KRAS wt, metastatic colorectal cancer: a pilot phase II trial. Br J Cancer. 2012;107:1932-1937.

126. Douillard JY, Siena S, J. Cassidy J, et al. Final results from PRIME: randomized phase III study of panitumumab with FOLFOX4 for firstline treatment of metastatic colorectal cancer. Ann Oncol. 2014;25: 1346-1355.

127. Fornaro L, Lonardi S, Masi G, et al. FOLFOXIRI in combination with panitumumab as first-line treatment in quadruple wild-type (KRAS, NRAS, HRAS, BRAF) metastatic colorectal cancer patients: a phase II trial by the Gruppo Oncologico Nord Ovest (GONO). Ann Oncol. 2013;24:2062-2067.

128. Nordlinger B, Sorbye H, Glimelius B, et al. Perioperative chemotherapy with FOLFOX4 and surgery versus surgery alone for resectable liver metastases from colorectal cancer (EORTC Intergroup trial 40983): a randomised controlled trial. Lancet. 2008;371: 1007-1016.

129. Primrose J, Falk S, Finch-Jones M, et al. Systemic chemotherapy with or without cetuximab in patients with resectable colorectal liver metastasis: the New EPOC randomised controlled trial. Lancet Oncol. 2014;15:601-611.

130. Bennouna J, Sastre J, Arnold D, et al. Continuation of bevacizumab after first progression in metastatic colorectal cancer (ML18147): a randomised phase 3 trial. Lancet Oncol. 2013;14:29-37.

131. Peeters M, Price TJ, Cervantes A, et al. Randomized phase III study of panitumumab with fluorouracil, leucovorin, and irinotecan (FOLFIRI) compared with FOLFIRI alone as second-line treatment in patients with metastatic colorectal cancer. J Clin Oncol. 2010;28:4706-4713.

132. Ciardiello F, Maiello E, Pisconti S, et al. Optimal treatment strategy in KRAS wild type (wt) metastatic colorectal cancer (mCRC): Cetuximab plus FOLFIRI followed by FOLFOX4 with or without cetuximabThe Capri trial from the Gruppo Oncologico Dell'Italia Meridionale (GOIM). J Clin Oncol. 2013;31(suppl): abstr e14565.

133. Sobrero A, Maurel J, Fehrenbacher L, et al. EPIC: phase III trial of cetuximab plus irinotecan after fluoropyrimidine and oxaliplatin failure in patients with metastatic colorectal cancer. J Clin Oncol. 2008;26:2311-2319.

134. Jonker DJ, O'Callaghan CJ, Karapetis CS, et al. Cetuximab for the treatment of colorectal cancer. N Engl J Med. 2007;357: 2040-2048.

135. Van Cutsem E, Peeters M, Siena S, et al. Open-label phase III trial of panitumumab plus best supportive care compared with best supportive care alone in patients with chemotherapy-refractory metastatic colorectal cancer. J Clin Oncol. 2007;25:1658-1664.

136. Price TJ, Peeters M, Kim TW, et al. Panitumumab versus cetuximab in patients with chemotherapy-refractory wild-type KRAS exon 2 metastatic colorectal cancer (ASPECCT): a randomised, multicentre, open-label, non-inferiority phase 3 study. Lancet Oncol. 2014;15:569-579.

137. Santini D, Loupakis F, Vincenzi B, et al. High concordance of KRAS status between primary colorectal tumors and related metastatic sites: implications for clinical practice. Oncologist. 2008;13:1270-1275.

138. Gattenlöhner S, Etschmann B, Kunzmann V, et al. Concordance of KRAS/BRAF mutation status in metastatic colorectal cancer before and after anti-EGFR therapy. J Oncol. 2009;2009:831626.

139. Santini D, Vincenzi B, Addeo R, et al. Cetuximab rechallenge in metastatic colorectal cancer patients: how to come away from acquired resistance? Ann Oncol. 2012;23:2313-2318.

140. Metges J, Raoul J, Achour N, et al. PANERB study: panitumumab after cetuximab-based regimen failure. J Clin Oncol. 2010;28:e140000.

141. Ciardiello F, Bianco R, Damiano V, et al. Antiangiogenic and antitumor activity of anti-epidermal growth factor receptor C225 monoclonal antibody in combination with vascular endothelial growth factor antisense oligonucleotide in human GEO colon cancer cells. Clin Cancer Res. 2000;6:3739-3747. 
142. Shaheen RM, Ahmad SA, Liu W, et al. Inhibited growth of colon cancer carcinomatosis by antibodies to vascular endothelial and epidermal growth factor receptors. Br J Cancer. 2001;85:584-589.

143. Jung YD, Mansfield PF, Akagi M, et al. Effects of combination antivascular endothelial growth factor receptor and anti-epidermal growth factor receptor therapies on the growth of gastric cancer in a nude mouse model. Eur J Cancer. 2002;38:1133-1140.

144. Tonra JR, Deevi DS, Corcoran E, et al. Synergistic antitumor effects of combined epidermal growth factor receptor and vascular endothelial growth factor receptor-2 targeted therapy. Clin Cancer Res. 2006;12:2197-2207.

145. Saltz LB, Lenz HJ, Kindler HL, et al. Randomized phase II trial of cetuximab, bevacizumab, and irinotecan compared with cetuximab and bevacizumab alone in irinotecan-refractory colorectal cancer: the BOND-2 study. J Clin Oncol. 2007;25:4557-4561.

146. Hecht JR, Mitchell E, Chidiac T, et al. A randomized phase IIIB trial of chemotherapy, bevacizumab, and panitumumab compared with chemotherapy and bevacizumab alone for metastatic colorectal cancer. J Clin Oncol. 2009;27:672-680.

147. Adams RA, Meade AM, Seymour MT, et al. Intermittent versus continuous oxaliplatin and fluoropyrimidine combination chemotherapy for first-line treatment of advanced colorectal cancer: results of the randomised phase 3 MRC COIN trial. Lancet Oncol. 2011;12: 642-653.

148. Wasan H, Meade AM, Adams R, et al. Intermittent chemotherapy plus either intermittent or continuous cetuximab for first-line treatment of patients with KRAS wild-type advanced colorectal cancer (COIN-B): a randomised phase 2 trial. Lancet Oncol. 2014;15: 631-639.

149. Pedersen MW, Jacobsen HJ, Koefoed K, et al. Sym004: a novel synergistic anti-epidermal growth factor receptor antibody mixture with superior anticancer efficacy. Cancer Res. 2010;70: 588-597.

150. Dienstmann R, Tabernero J, Van Cutsem E, et al. Proof-of-concept study of Sym004, an anti-EGFR monoclonal antibody (mAb) mixture, in patients (pts) with anti-EGFR mab-refractory KRAS wildtype (wt) metastatic colorectal cancer (mCRC). J Clin Oncol. 2013; Suppl 31:3551.

151. EMD Serono. Sym004 vs Standard of Care in Subjects With Metastatic Colorectal Cancer. Available from http://clinicaltrial.gov/ct2/ show/NCT02083653. NLM identifier: NCT02083653. Accessed November 14, 2014.

152. Baselga J, Swain SM. Novel anticancer targets: revisiting ERBB2 and discovering ERBB3. Nat Rev Cancer. 2009;9:463-475.

153. Scartozzi M, Mandolesi A, Giampieri R, et al. The role of HER-3 expression in the prediction of clinical outcome for advanced colorectal cancer patients receiving irinotecan/cetuximab. J Clin Oncol. 2011; 29 Suppl 4:404.

154. Cervantes-Ruiperez A, Juric D, Hidalgo M, et al. A phase I study of MEHD7945A (MEHD), a first-in-class HER3/EGFR dual-action antibody, in patients (pts) with refractory/recurrent epithelial tumors: expansion cohorts. J Clin Oncol. 2012;Suppl 30:2568.

155. Yonesaka K, Zejnullahu K, Okamoto I, et al. Activation of ERBB2 signaling causes resistance to the EGFR-directed therapeutic antibody cetuximab. Sci Transl Med. 2011;7:99ra86.

156. Bertotti A, Migliardi G, Galimi F, et al. A molecularly annotated platform of patient-derived xenografts ("xenopatients") identifies HER2 as an effective therapeutic target in cetuximab-resistant colorectal cancer. Cancer Discov. 2011;1:508-523.

157. Martin V, Landi L, Molinari F, et al. HER2 gene copy number status may influence clinical efficacy to anti-EGFR monoclonal antibodies in metastatic colorectal cancer patients. $\mathrm{Br} J$ Cancer. 2013;108:668-675.

158. NSABP Foundation Inc. Safety and Efficacy Study of Neratinib and Cetuximab to Treat Patients With Quadruple Wild-Type Metastatic Colorectal Cancer. Available from: http://clinicaltrials.gov/ct2/show/NCT01960023. NLM identifier: NCT01960023. Accessed November 14, 2014.
159. Reidy DL, Vakiani E, Fakih MG, et al. Randomized, phase II study of the insulin-like growth factor-1 receptor inhibitor IMCA12, with or without cetuximab, in patients with cetuximab- or panitumumab-refractory metastatic colorectal cancer. J Clin Oncol. 2010;28:4240-4246.

160. Watkins DJ, Tabernero J, Schmoll H, et al. A randomized phase II/III study of the anti-IGF-1R antibody MK-0646 (dalotuzumab) in combination with cetuximab $(\mathrm{Cx})$ and irinotecan (Ir) in the treatment of chemorefractory metastatic colorectal cancer (mCRC) with wild-type (wt) KRAS status. J Clin Oncol. 2011;Suppl 29:3501.

161. Eng C, Hart L, Severtsev S, et al. A randomized, placebo-controlled, phase I/II study of tivantinib (ARQ 197) in combination with cetuximab and irinotecan in patients (pts) with KRAS wild-type (WT) metastatic colorectal cancer (CRC) who had received previous frontline systemic therapy. J Clin Oncol. 2013;Suppl 31:3508.

162. Van Cutsem E, Eng C, Nowara E, et al. Randomized phase Ib/II trial of rilotumumab or ganitumab with panitumumab versus panitumumab alone in patients with wild-type KRAS metastatic colorectal cancer. Clin Cancer Res 2014;20:4240-4250.

163. Peeters M, Infante JR, Rougier P et al. Phase Ib/II trial of conatumumab and panitumumab (pmab) for the treatment (tx) of metastatic colorectal cancer (mCRC): Safety and efficacy. J Clin Oncol. 2010; Suppl 27:443.

164. Taber AM, Khurshid H, Perez K, et al. Phase I study of ridaforolimus with cetuximab for patients with advanced non-small cell lung cancer (NSCLC), colorectal cancer, and head and neck cancer. J Clin Oncol. 2013;Supp1 31:8075.

165. Deming DA, Schelman W, Lubner J, et al. A phase I study of selumetinib (AZD6244/ARRY-142866) in combination with cetuximab (cet) in refractory solid tumors and KRAS mutant colorectal cancer (CRC). J Clin Oncol. 2012;Suppl 30:3103.

166. Bristol-Myers Squibb. Combination Study of BMS-754807 and Erbitux ${ }^{\circledR}$ in Subjects With Advanced or Metastatic Solid Tumors. Available from: http://clinicaltrials.gov/show/ NCT00908024. NLM identifier: NCT00908024. Accessed November 14, 2014.

167. Eli Lilly and Company. A study of LY2801653 in Advanced Cancer. Available from: http://clinicaltrials.gov/ct2/show/ NCT01285037. NLM identifier: NCT01285037. Accessed November 14, 2014.

168. Merck KGaA. EMD 525797 in Combination With Cetuximab and Irinotecan in K-ras Wildtype Metastatic Colorectal Cancer (POSEIDON). Available from: http://clinicaltrials.gov/ct2/ show/NCT01008475. NLM identifier: NCT01008475. Accessed November 14, 2014.

169. National Cancer Institute (NCI). BAY 43-9006 Plus Cetuximab to Treat Colorectal Cancer. Available from: http://clinicaltrials.gov/ ct2/show/NCT00326495. NLM identifier: NCT00326495. Accessed November 14, 2014.

170. Massachusetts General Hospital. Temsirolimus (CCI-770, Torisel) Combined With Cetuximab in Cetuximab-Refractory Colorectal Cancer. Available from: http://clinicaltrials.gov/ct2/ show/NCT00593060. NLM identifier: NCT00593060. Accessed November 14, 2014.

171. Hoosier Cancer Research Network. Phase I/Randomized Phase II Study of Second Line Therapy With Irinotecan + Cetuximab +/- RAD001 for Colorectal Cancer. Available from: http://clinicaltrials.gov/ct2/ show/NCT00522665. NLM identifier: NCT00522665. Accessed November 14, 2014.

172. Oncothyreon Inc. Phase 1 and 2 Study of PX-866 and Cetuximab. Available from: http://clinicaltrials.gov/ct2/show/NCT01252628. NLM identifier: NCT01252628. Accessed November 14, 2014.

173. Pfizer. A Study Of PF-05212384 Plus Irinotecan Vs Cetuximab Plus Irinotecan In Patients With KRAS And NRAS Wild Type Metastatic Colorectal Cancer. Available from: http://clinicaltrials.gov/ct2/ show/NCT01925274. NLM identifier: NCT01925274. Accessed November 14, 2014. 
174. Novartis Pharmaceuticals. Study of LGX818 and Cetuximab or LGX818, BYL719, and Cetuximab in BRAF Mutant Metastatic Colorectal Cancer. Available from: http:/clinicaltrials.gov/ct2/ show/NCT01719380. NLM identifier: NCT01719380. Accessed November 14, 2014.

175. Hoffmann-La Roche. A Study of Zelboraf (Vemurafenib) in Patients With BRAF V600 Mutation-Positive Cancers. Available from: http:/clinicaltrials.gov/ct2/show/NCT01524978. NLM identifier: NCT01524978. Accessed November 14, 2014.
176. GlaxoSmithKline. BRAF/MEK/EGFR Inhibitor Combination Study in Colorectal Cancer. Available from: http://clinicaltrials.gov/ct2/ show/NCT01750918. NLM identifier: NCT01750918. Accessed November 14, 2014.

177. Novartis Pharmaceuticals. Phase Ib/II Study of Efficacy and Safety of MEK162 and Panitumumab, in Adult mCRC Patients With Mutant or Wild-type RAS Tumors. Available from: http:/clinicaltrials.gov/ ct2/show/NCT01927341. NLM identifier: NCT01927341. Accessed November 14, 2014.

\section{Publish your work in this journal}

Gastrointestinal Cancer: Targets and Therapy is an international, peer-reviewed, open access journal focusing on gastro-intestinal cancer research, identification of therapeutic targets and the optimal use of preventative and integrated treatment interventions to achieve improved outcomes, enhanced survival and quality of life for the cancer patient. The manuscript management system is completely online and includes a very quick and fair peer-review system. Visit http://www.dovepress.com/testimonials.php to read real quotes from published authors.

Submit your manuscript here: http://www.dovepress.com/gastro-intestinal-cancer-targets-and-therapy-journal 